\title{
A Diffusion Model for Rarefied Flows in Curved Channels
}

$\operatorname{AUTHOR}(S)$ :

Aoki, K.; Degond, P.; Mieussens, L.; Takata, S.; Yoshida, $\mathrm{H}$.

\section{CITATION:}

Aoki, K....[et al]. A Diffusion Model for Rarefied Flows in Curved Channels. Multiscale Modeling \& Simulation 2008, 6(4): 1281-1316

\section{ISSUE DATE:}

2008-02

URL:

http://hdl.handle.net/2433/85229

RIGHT:

Copyright (c) 2008 Society for Industrial and Applied Mathematics 


\title{
A DIFFUSION MODEL FOR RAREFIED FLOWS IN CURVED CHANNELS*
}

\author{
K. AOKI $^{\dagger}$, P. DEGOND ${ }^{\ddagger}$, L. MIEUSSENS ${ }^{\ddagger}$, S. TAKATA $^{\dagger}$, AND H. YOSHIDA $^{\dagger}$
}

\begin{abstract}
In this paper, we derive a one-dimensional convection-diffusion model for a rarefied gas flow in a two-dimensional curved channel on the basis of the Boltzmann (Bhatnagar-GrossKrook) model. The flow is driven by the temperature gradient along the channel walls, which is known as the thermal creep phenomenon. This device can be used as a micropumping system without any moving part. Our derivation is based on the asymptotic technique of the diffusion approximation. It gives a macroscopic (fluid) approximation of the microscopic (kinetic) equation. We also derive the connection conditions at the junction where the curvature is not continuous. The pumping device is simulated by using a numerical approximation of our convection-diffusion model which turns out to agree very well with full two-dimensional kinetic simulations. It is then used to obtain very fast computations on long pumping devices, while the computational cost of full kinetic computations nowadays is still prohibitive for such cases.
\end{abstract}

Key words. Boltzmann equation, rarefied gas flows, diffusion approximation, convectiondiffusion model, Knudsen compressor, thermal creep flow

AMS subject classifications. 76P05, 82B40, 41A60, 76R05, 82C80, 65M06

DOI. $10.1137 / 070690328$

1. Introduction. Recently, the possibility of making a pumping system for a rarefied gas in a curved channel has been proposed by Aoki, Degond, and Mieussens [2] and Aoki et al. [3]. This pumping effect is generated by the well-known thermal creep flow (also known as thermal transpiration in this case), which is caused only by a temperature gradient applied to the walls of the channel. This phenomenon has been known since Reynolds [29]. It was first used by Knudsen [22] to design what is now called a Knudsen compressor. The technological advantage of such a pumping system is that it uses no moving part nor mixing process but only this thermal creep boundary effect that exists only for rarefied gases. It can be used for gases at atmospheric pressure if the size of the system is small enough. This is why recently interest in this kind of flow is growing in connection with micromachine engineering, such as in microelectromechanical systems (MEMS).

The system proposed in $[2,3]$ uses a periodic temperature distribution of the channel walls together with a change of their curvature. It is similar to a system proposed in $[41,5,38]$, but its geometry is simpler. It has been proved by numerical simulations that this system can actually produce a pumping effect that makes it technologically promising. However, large numerical simulations of rarefied gas problems are still a delicate issue, since this always implies using a large number of degrees of

*Received by the editors May 2, 2007; accepted for publication (in revised form) November 9, 2007; published electronically February 22, 2008. This research was partially supported by the joint research project between JSPS and CNRS "Projet International de Coopération Scientifique (PICS)" of CNRS, grant 3195 and grants-in-aid for scientific research from JSPS (17360041), and from MEXT (1765033); computational resources were provided by the CALMIP project (Calcul en Midi-Pyrénées), University Paul Sabatier, Toulouse, France.

http://www.siam.org/journals/mms/6-4/69032.html

${ }^{\dagger}$ Department of Mechanical Engineering and Science, Graduate School of Engineering, Kyoto University, Kyoto 606-8501, Japan (aoki@aero.mbox.media.kyoto-u.ac.jp, takata@aero.mbox.media. kyoto-u.ac.jp, hiroaki.yos@gmail.com).

‡Institut de Mathématiques, Université de Toulouse et CNRS (UMR 5219), 31062 Toulouse cedex 9, France (degond@mip.ups-tlse.fr, mieussens@mip.ups-tlse.fr). 
freedom. Indeed, even for two-dimensional plane flows, the distribution function of the particle velocities of the gas has six independent variables. This is why in $[2,3]$ the length of the channel is quite limited, which also limits the study of the device to relatively small pressure gains.

The purpose of this paper then is to derive a macroscopic model for the gas density in a section of the channel when the width of this section is small as compared to its length. The computational complexity of the problem is largely reduced, since this density has only two independent variables. This macroscopic model, also called fluid approximation, is a one-dimensional nonlinear convection-diffusion equation. However, the transport and diffusion coefficients are not given by analytical formula but are tabulated once for all by solving auxiliary linear one-dimensional steady kinetic problems.

This kind of derivation has first been rigorously proposed by Babovsky, Bardos, and Płatkowski in [7] for free molecular flows and relies on the previous work by Babovsky [6]. These works have recently been used by Aoki and Degond [1] to formally derive a diffusion model for the Knudsen pump studied in [41, 5]. The method is based on a diffusion approximation technique, using the boundary scattering operator as the forcing term towards equilibrium. Here we adapt the work of [1] to the case of our curved channel. The major differences with [1] are the following. First, the geometry of our channel requires using a curvilinear coordinate system, which makes the transport operator more complicated. Second, the collision operator we consider in this work is the Bhatnagar-Gross-Krook (BGK) model of the Boltzmann equation. While this simplification is not physically well justified, it allows us to easily reduce the computational complexities of collisions. Moreover, it is useful for obtaining qualitative information on a rarefied flow, and it is known to give accurate results in some situations [16]. In [1], the collision model was only a simplified version of the BGK model in which the distribution function is assumed to relax to a Maxwellian with zero mean velocity and the same temperature as the wall temperature. However, we mention that an extension of [1] to the Boltzmann and the full BGK models can be found in [4]. This approach has also been applied to gaseous mixtures [44]. Third, our diffusion model is used for various numerical simulations, and our approach is validated by comparisons with full kinetic simulations. Two different systems are used for this validation: a ring-shaped channel in which we simulate a circulating flow, and a cascade system closed at both ends for which the pumping effect is demonstrated. Then the potentiality of our diffusion model is shown by an application to a very large cascade system of 100 units for which full kinetic simulations would at present require prohibitive computational time and memory.

Note that the geometry of our device is such that the curvature of the channel is discontinuous at some points: this is taken into account by a kinetic transmission layer analysis that was already used in [1]. It should also be stressed that, although the diffusion model is applied only to the pump mentioned above in the present paper, it has potential applicability to general gas flows through a curved channel.

Before we give the scope of the paper, we present a rapid bibliography about the thermal creep flow phenomenon and its application to pumping systems. The reader is referred to, e.g., [21, 32, 42, 27] for the thermal creep flow. Several analyses of temperature induced flows in rarefied gases can be found in Sone [33, 34, 35]. A valuable source of information about flows in micromachines can be found in Karniadakis, Beskok, and Aluru [20] and Cercignani [10]. Examples of experimental and simulation works on Knudsen compressors and MEMS have been given by Pham-Van-Diep et al. [28], Sone, Waniguchi, and Aoki [41], Aoki et al. [5], Vargo and Muntz [45], Sone 


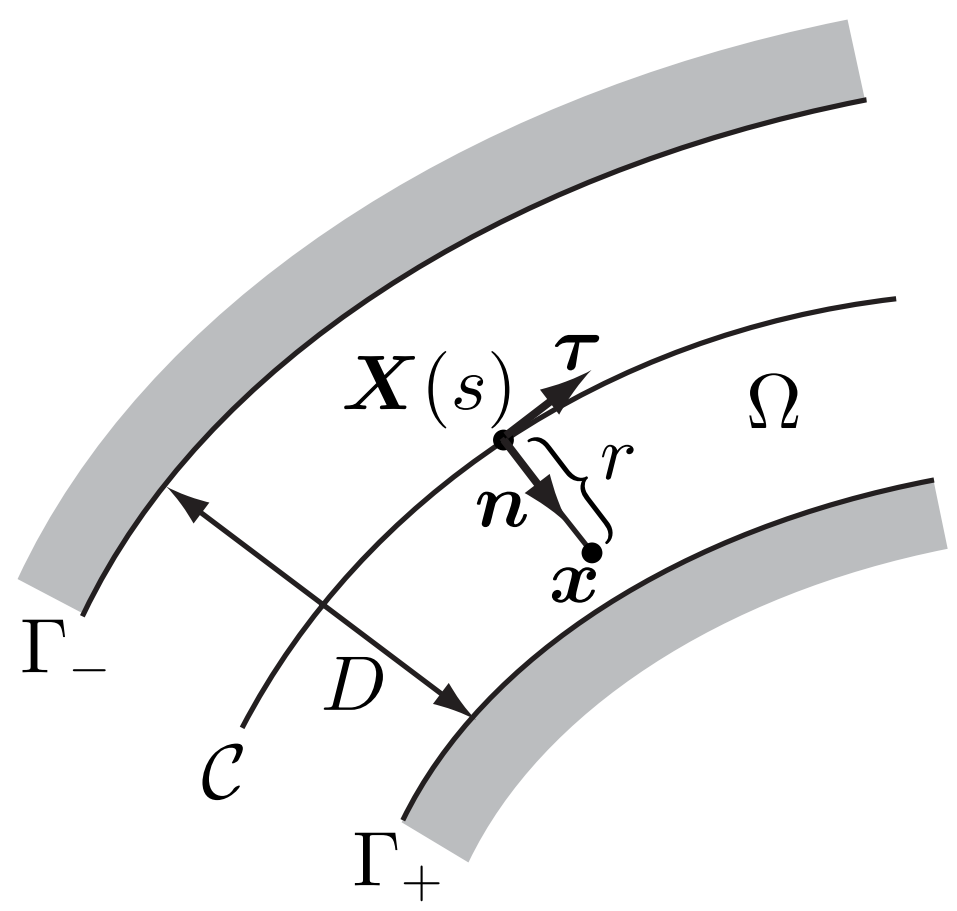

FIG. 1. Two-dimensional curved channel $\Omega$ and the curvilinear coordinate system.

and Sugimoto [39], and Han et al. [18].

The outline of the paper is the following. In section 2, the kinetic model is presented both in Cartesian and curvilinear coordinates. After a dimensional analysis, the asymptotic diffusion model is derived, and some formal justifications are given. The junction problem for discontinuous curvature is also studied in section 3. In section 4 , we give some information about the numerical methods we use to approximate the diffusion model. Finally, we present our simulation results in section 5 .

\section{Derivation of a diffusion model for rarefied flows in a curved channel.}

2.1. Kinetic model. We consider a rarefied flow in a two-dimensional curved channel $\Omega$ of constant width $D$. To describe the problem, we introduce the following coordinate system. The two-dimensional position vector of the median curve $\mathcal{C}$ of the channel is parameterized by the curve length $s$ and denoted by $\boldsymbol{X}(s)$, which is supposed to be smooth. We denote the curvature of $\mathcal{C}$ by $\kappa(s)$, the unit tangent vector (in the positive direction of $s$ ) by $\boldsymbol{\tau}(s)=d \boldsymbol{X} / d s$, and the unit normal vector by $\boldsymbol{n}(s)$ such that the frame $(\boldsymbol{\tau}, \boldsymbol{n})$ is direct (see Figure 1). By Frenet's formula, the following relations hold:

$$
d \boldsymbol{\tau} / d s=\kappa \boldsymbol{n}, \quad d \boldsymbol{n} / d s=-\kappa \boldsymbol{\tau} .
$$

We introduce the following curvilinear coordinate system associated with $\mathcal{C}$ :

$$
\boldsymbol{x}=\boldsymbol{X}(s)+r \boldsymbol{n} .
$$

For $\left(x_{1}, x_{2}\right) \in \mathbb{R}^{2}$ sufficiently close to $\mathcal{C}$, the mapping $\mathcal{F}:\left(x_{1}, x_{2}\right) \rightarrow(s, r) \in \mathbb{R}^{2}$ is an admissible change of variables. More precisely, we assume that $\mathcal{F}$ is a $C^{\infty}$ 
diffeomorphism from an open neighborhood $\Omega$ of $\mathcal{C}$ into $\mathbb{R} \times(-D / 2, D / 2)$. The twodimensional volume element in this new system of coordinates is given by $d \boldsymbol{x}=$ $(1-\kappa r) d s d r$, and the Jacobian $(1-\kappa r)$ is supposed to be positive.

The temperature of both walls is kept at $T_{w}(s)$. Let $\left(x_{1}, x_{2}, x_{3}\right)$ denote the Cartesian coordinates of a point $\boldsymbol{x} \in \Omega$. Physically, $\Omega$ describes a three-dimensional domain which has translational invariance in a third direction $x_{3}$ orthogonal to $\left(x_{1}, x_{2}\right)$. Particles moving in such a domain do in general have a nonzero velocity component along this third coordinate, and we are led to consider three-dimensional velocity vectors $\boldsymbol{v}=\left(v_{1}, v_{2}, v_{3}\right)$. Now let $f(\boldsymbol{x}, \boldsymbol{v}, t)$ denote the distribution function of the particles at position $\boldsymbol{x} \in \Omega$ and time $t>0$ with velocity $\boldsymbol{v} \in \mathbb{R}^{3}$.

While in the rarefied regime $f$ should be governed by the Boltzmann equation, we consider here the simpler BGK relaxation model $[8,46]$. The BGK collision operator is homogeneous of degree 2, like the Boltzmann operator, but it leads to much simpler asymptotic computations. The BGK equation reads

$$
\partial_{t} f+\boldsymbol{v} \cdot \nabla_{\boldsymbol{x}} f=A_{c} \rho(M[\rho, \boldsymbol{u}, 2 R T]-f),
$$

where $A_{c}$ is a constant (physically, $A_{c} \rho$ corresponds to the collision frequency), $M[\rho, \boldsymbol{u}$, $2 R T]$ is the Maxwellian equilibrium distribution

$$
M[\rho, \boldsymbol{u}, 2 R T]=\frac{\rho}{(2 \pi R T)^{3 / 2}} \exp \left(-\frac{(\boldsymbol{v}-\boldsymbol{u})^{2}}{2 R T}\right),
$$

and $\rho, \boldsymbol{u}=\left(u_{s}, u_{r}, 0\right)$, and $T$ are, respectively, the density, the flow velocity, and the temperature of the gas defined by

$$
\rho=\int f d \boldsymbol{v}, \quad \rho \boldsymbol{u}=\int \boldsymbol{v} f d \boldsymbol{v}, \quad 3 R \rho T=\int(\boldsymbol{v}-\boldsymbol{u})^{2} f d \boldsymbol{v} .
$$

Finally, $R$ is the gas constant per unit mass, i.e., $R=k_{B} / m$, where $k_{B}$ is the Boltzmann constant and $m$ is the mass of a gas molecule.

By the translational invariance in the direction $x_{3}$, we have $\boldsymbol{v} \cdot \nabla_{\boldsymbol{v}} f=v_{1} \partial_{x_{1}} f+$ $v_{2} \partial_{x_{2}} f$, and it can be assumed that there is no macroscopic velocity along $x_{3}$, namely $u_{3}=0$.

The interaction of the gas with the boundaries $\Gamma_{ \pm}$(see Figure 1) is classically assumed to be well described by the following diffuse reflection:

$$
f\left(\boldsymbol{x} \in \Gamma_{ \pm}, \boldsymbol{v}, t\right)=M\left[1,0,2 R T_{w}(\boldsymbol{x})\right] \frac{\int_{\boldsymbol{v} \cdot \boldsymbol{n} \gtrless 0}|\boldsymbol{v} \cdot \boldsymbol{n}| f d \boldsymbol{v}}{\int_{\boldsymbol{v} \cdot \boldsymbol{n} \lessgtr 0}|\boldsymbol{v} \cdot \boldsymbol{n}| M\left[1,0,2 R T_{w}(\boldsymbol{x})\right] d \boldsymbol{v}} \quad \text { for } \boldsymbol{v} \cdot \boldsymbol{n} \lessgtr 0 .
$$

This means that molecules hitting the walls are re-emitted along a Maxwellian velocity distribution with a temperature equal to that of the wall and with zero mean velocity. The integral factor implies that all the particles are re-emitted; that is, there is no mass flux across the walls. Finally, the BGK equation (2.3) must also be supplemented by an initial condition $f_{I}=f(\boldsymbol{x}, \boldsymbol{v}, t=0)$.

Now we write the BGK equation in the curved coordinate system $(r, s)$. We introduce a local velocity coordinate system $\left(v_{s}, v_{r}\right)$ according to

$$
v_{s}=\boldsymbol{v} \cdot \boldsymbol{\tau}, \quad v_{r}=\boldsymbol{v} \cdot \boldsymbol{n} .
$$

Denoting again by $f\left(s, r, v_{s}, v_{r}, v_{3}, t\right)$ the distribution $f$ in these new variables, we have the following lemma.

LEMMA 2.1. In the curved coordinate system $(s, r),(2.3)$ is written in the following form: 


$$
\begin{aligned}
\partial_{t} f+(1-\kappa r)^{-1} v_{s} \partial_{s} f+v_{r} \partial_{r} f & +\kappa(1-\kappa r)^{-1} v_{r} v_{s} \partial_{v_{s}} f \\
& -\kappa(1-\kappa r)^{-1} v_{s}^{2} \partial_{v_{r}} f=A_{c} \rho(M[\rho, \boldsymbol{u}, 2 R T]-f) .
\end{aligned}
$$

The diffuse-reflection boundary condition is written as

$$
f= \pm \frac{1}{2 \pi\left(R T_{w}\right)^{2}} \exp \left(-\frac{\boldsymbol{v}^{2}}{2 R T_{w}}\right) \int_{v_{r} \gtrless 0} v_{r} f d \boldsymbol{v} \quad \text { for } v_{r} \lessgtr 0 \quad \text { at } r= \pm \frac{D}{2} .
$$

Proof. Straightforward differential geometry shows that

$$
\nabla_{x} s=(1-\kappa r)^{-1} \boldsymbol{\tau}, \quad \nabla_{x} r=\boldsymbol{n} .
$$

Taking the gradients w.r.t. $x$ of (2.5) and using (2.8) leads to

$$
\nabla_{x} v_{s}=\kappa(1-\kappa r)^{-1} v_{r} \boldsymbol{\tau}, \quad \nabla_{x} v_{r}=-\kappa(1-\kappa r)^{-1} v_{s} \boldsymbol{\tau} .
$$

Using (2.8), (2.9), and the chain rule leads to (2.6). The other equations are straightforward.

Now, in order to identify the important parameters of the problem, we make a dimensional analysis of the equation. We denote by $L_{s}$ the typical length scale of variation of $f$ in the $s$ direction. In other words, the scale of variation of $T_{w}$ and $\kappa$ is $L_{s}$. We suppose that $L_{s}$ is much longer than the channel width $D$, i.e., $\epsilon=D / L_{s} \ll 1$. Keeping this in mind, we introduce the following dimensionless variables:

$$
\begin{gathered}
t^{\prime}=\frac{t}{t_{0}}, \quad r^{\prime}=\frac{r}{D}, \quad s^{\prime}=\frac{s}{L_{s}}, \quad \boldsymbol{v}^{\prime}=\frac{\boldsymbol{v}}{\left(2 R T_{0}\right)^{1 / 2}}, \quad f^{\prime}=\frac{\left(2 R T_{0}\right)^{3 / 2}}{\rho_{0}} f, \\
\rho^{\prime}=\frac{\rho}{\rho_{0}}, \quad \boldsymbol{u}^{\prime}=\frac{\boldsymbol{u}}{\left(2 R T_{0}\right)^{1 / 2}}, \quad T^{\prime}=\frac{T}{T_{0}}, \quad T_{w}^{\prime}=\frac{T_{w}}{T_{0}}, \quad \kappa^{\prime}=D \kappa,
\end{gathered}
$$

where $t_{0}, T_{0}$, and $\rho_{0}$ are the reference time, temperature, and the reference density, respectively; in the present study, we choose the following time scale as the reference time:

$$
t_{0}=\frac{L_{s}^{2}}{D\left(2 R T_{0}\right)^{1 / 2}}\left[=\frac{L_{s}}{\epsilon\left(2 R T_{0}\right)^{1 / 2}}\right] .
$$

This choice of time scale corresponds to equating the Strouhal number defined by Sh $=D / t_{0}\left(2 R T_{0}\right)^{1 / 2}$ with $\epsilon^{2}$; it will be justified a posteriori at the end of section 2.2.1. With these variables, the BGK equation (2.6) is rewritten as (from now on, we drop the superscript ' for simplicity)

$$
\begin{aligned}
\epsilon^{2} \partial_{t} f+\epsilon(1-\kappa r)^{-1} v_{s} \partial_{s} f & +v_{r} \partial_{r} f+\kappa(1-\kappa r)^{-1} v_{r} v_{s} \partial_{v_{s}} f \\
& -\kappa(1-\kappa r)^{-1} v_{s}^{2} \partial_{v_{r}} f=\frac{1}{K_{0}} \rho(M[\rho, \boldsymbol{u}, T]-f),
\end{aligned}
$$

where according to definition (2.4) we have

$$
M[\rho, \boldsymbol{u}, T]=\frac{\rho}{(\pi T)^{3 / 2}} \exp \left(-\frac{(\boldsymbol{v}-\boldsymbol{u})^{2}}{T}\right),
$$

and the macroscopic quantities now are defined by

$$
\rho=\int f d \boldsymbol{v}, \quad \rho \boldsymbol{u}=\int \boldsymbol{v} f d \boldsymbol{v}, \quad \frac{3}{2} \rho T=\int(\boldsymbol{v}-\boldsymbol{u})^{2} f d \boldsymbol{v} .
$$

Copyright (C) by SIAM. Unauthorized reproduction of this article is prohibited. 
Finally, $K_{0}$ is the Knudsen number defined by

$$
K_{0}=\frac{\sqrt{\pi}}{2} \frac{\ell_{0}}{D}=\frac{\left(2 R T_{0}\right)^{1 / 2}}{D A_{c} \rho_{0}},
$$

where $\ell_{0}$ is the mean free path of the gas at equilibrium state at rest with temperature $T_{0}$ and density $\rho_{0}$. The boundary condition (2.7) is rewritten as

$$
f= \pm \frac{2}{\pi T_{w}^{2}} \exp \left(-\frac{\boldsymbol{v}^{2}}{T_{w}}\right) \int_{v_{r} \gtrless 0} v_{r} f d \boldsymbol{v} \quad \text { for } v_{r} \lessgtr 0 \quad \text { at } r= \pm \frac{1}{2} .
$$

It is clear that if the temperature distribution $T_{w}$ and curvature $\kappa$ of the channel walls are given, problem (2.13)-(2.17) depends only on two parameters $K_{0}$ and $\epsilon$. In what follows, we are interested in the limit $\epsilon \rightarrow 0$ of this problem.

2.2. Diffusion model for smoothly varying curvature. Classically, an asymptotic model for (2.13) will necessarily be a macroscopic relation based on a conservation law. Since we are interested in a limit where the width of the channel becomes small, it is natural to expect an asymptotic model of the form of an integrated conservation law in a section of the channel. Consequently, we first write such a conservation law for a fixed $\epsilon$.

LEMMA 2.2. For every $\epsilon>0$, we have the continuity equation

$$
\partial_{t} \varrho+\partial_{s} j=0,
$$

with

$$
\begin{aligned}
& \varrho(s, t)=\int_{-1 / 2}^{1 / 2} \int_{\mathbb{R}^{3}} f(1-\kappa r) d \boldsymbol{v} d r, \\
& j(s, t)=\frac{1}{\epsilon} \int_{-1 / 2}^{1 / 2} \int_{\mathbb{R}^{3}} v_{s} f d \boldsymbol{v} d r .
\end{aligned}
$$

Proof. This relation is simply obtained by multiplying the BGK equation (2.13) by the Jacobian $(1-\kappa r)$ of the transformation $\left(x_{1}, x_{2}\right) \rightarrow(s, r)$ and then by integrating in the velocity space $\mathbb{R}^{3}$ and in a section of the channel.

Equation (2.18) is the continuity equation which expresses that the time variation of the total mass of gas in a given section of the channel must be balanced by the difference of the fluxes through its boundaries.

In these relations, $\varrho$ is the gas density in a section of the channel, and $j$ is the total mass flux in the $s$-direction scaled by $\epsilon$. This scaling is due to the time scale chosen in (2.10) and is necessary to obtain a nontrivial dynamics in the asymptotic limit $\epsilon \rightarrow 0$. Indeed, with a more natural time scale such as $t_{0}=L_{s} /\left(2 R T_{0}\right)^{1 / 2}$ (the time needed by a particle with a thermal velocity to travel on the typical length scale $\left.L_{s}\right)$, it can be seen that the mass flux vanishes in the limit $\epsilon \rightarrow 0$, which gives the trivial limit equation $\partial_{t} \varrho=0$. This shows that the time scale must be large enough to observe variations of the limit density.

Now we state in the following formal theorem what the asymptotic limit of (2.18) is.

TheOrem 2.3 (formal). (i) $f \rightarrow f_{(0)}=\rho_{(0)}(s, t) M\left[1,0, T_{w}(s)\right]$ as $\epsilon \rightarrow 0$, where $\rho_{(0)}(s, t)$ is a solution of the following nonlinear diffusion problem:

$$
\begin{aligned}
& \partial_{t} \rho_{(0)}+\partial_{s} j_{(1)}=0, \\
& j_{(1)}=\sqrt{T_{w}} M_{P} \partial_{s} \rho_{(0)}+\frac{\rho_{(0)}}{\sqrt{T_{w}}}\left(M_{P}+M_{T}\right) \partial_{s} T_{w},
\end{aligned}
$$

Copyright (C) by SIAM. Unauthorized reproduction of this article is prohibited. 
where $M_{P}$ and $M_{T}$ are nonlinear functions of $\rho_{(0)}$ defined through auxiliary linear kinetic equations (see the proof of the theorem).

(ii) The diffusion coefficient $M_{P}$ is negative.

(iii) $\rho_{(0)}$ and $j_{(1)}$ are formally second order approximations of the density $\varrho$ and current $j$ associated with $f$ :

$$
\varrho-\rho_{(0)}=O\left(\epsilon^{2}\right), \quad j-j_{(1)}=O\left(\epsilon^{2}\right) .
$$

Expression (2.22) shows that the temperature gradient acts as a force field with a mobility $\left(1 / \sqrt{T_{w}}\right)\left(M_{P}+M_{T}\right)$. The resulting flow is called the thermal creep flow or thermal transpiration flow (see, e.g., [35]).

For the proof of this theorem, we classically use the Hilbert procedure that can be summarized as follows: the solution $f$ is expanded as a power series of $\epsilon$ :

$$
f=f_{(0)}+\epsilon f_{(1)}+\epsilon^{2} f_{(2)}+\epsilon^{3} f_{(3)}+\cdots .
$$

Correspondingly, all the macroscopic quantities are also expanded:

$$
\begin{aligned}
& \rho=\rho_{(0)}+\rho_{(1)} \epsilon+\cdots, \\
& \boldsymbol{u}=\boldsymbol{u}_{(0)}+\boldsymbol{u}_{(1)} \epsilon+\cdots, \\
& T=T_{(0)}+T_{(1)} \epsilon+\cdots,
\end{aligned}
$$

The relations between $f_{(m)}$ and $\rho_{(m)}, \boldsymbol{v}_{(m)}$ and $T_{(m)}$, are summarized in Appendix A. Then these expansions are inserted into (2.13)-(2.17), and after using a Taylor expansion of the nonlinear terms, the coefficients $f_{(0)}, f_{(1)}, \ldots$ are determined so that terms of equal power in $\epsilon$ cancel. More precisely, if we identify the different operators corresponding to the different powers of $\epsilon$ in (2.13), we find the following four operators:

$$
\begin{aligned}
\text { order } \epsilon^{0}: \quad & \mathcal{A}^{0} f=v_{r} \partial_{r} f+\kappa(1-\kappa r)^{-1} v_{r} v_{s} \partial_{v_{s}} f-\kappa(1-\kappa r)^{-1} v_{s}^{2} \partial_{v_{r}} f, \\
& Q(f)=\frac{1}{K_{0}} \rho(M[\rho, \boldsymbol{u}, T]-f), \\
\text { order } \epsilon^{1}: & \mathcal{A}^{1} f=(1-\kappa r)^{-1} v_{s} \partial_{s} f, \\
\text { order } \epsilon^{2}: & \mathcal{A}^{2} f=\partial_{t} f .
\end{aligned}
$$

Then by injecting the series (2.23) into (2.13) and using the previous notations, we find

$(2.31)=O\left(\epsilon^{4}\right)$

$$
\begin{aligned}
& \mathcal{A}^{0} f_{(0)}-Q\left(f_{(0)}\right) \\
& +\epsilon\left(\mathcal{A}^{1} f_{(0)}+\mathcal{A}^{0} f_{(1)}-D Q\left(f_{(0)}\right)\left(f_{(1)}\right)\right) \\
& +\epsilon^{2}\left(\mathcal{A}^{2} f_{(0)}+\mathcal{A}^{1} f_{(1)}+\mathcal{A}^{0} f_{(2)}-D Q\left(f_{(0)}\right)\left(f_{(2)}\right)-\frac{1}{2} D^{2} Q\left(f_{(0)}\right)\left(f_{(1)}, f_{(1)}\right)\right) \\
& +\epsilon^{3}\left(\mathcal{A}^{2} f_{(1)}+\mathcal{A}^{1} f_{(2)}+\mathcal{A}^{0} f_{(3)}-D Q\left(f_{(0)}\right)\left(f_{(3)}\right)-D^{2} Q\left(f_{(0)}\right)\left(f_{(1)}, f_{(2)}\right)\right. \\
& \left.-\frac{1}{6} D^{3} Q\left(f_{(0)}\right)\left(f_{(1)}, f_{(1)}, f_{(1)}\right)\right)
\end{aligned}
$$

where $D Q\left(f_{(0)}\right)\left(f_{(1)}\right)$ denotes the first order derivative of $Q$ at $f_{(0)}$ applied to $f_{(1)}$, $D^{2} Q\left(f_{(0)}\right)\left(f_{(1)}, f_{(2)}\right)$ the second order derivative of $Q$ at $f_{(0)}$ applied to the pair 
$\left(f_{(1)}, f_{(2)}\right)$, and similarly for $D^{3} Q\left(f_{(0)}\right)\left(f_{(1)}, f_{(1)}, f_{(1)}\right)$. Accordingly, boundary condition (2.17) gives

$$
\sum_{m=0}^{3} \epsilon^{m} f_{(m)}=\sum_{m=0}^{3} \pm \frac{2}{\pi T_{w}^{2}} \exp \left(-\frac{\boldsymbol{v}^{2}}{T_{w}}\right) \int_{v_{r} \gtrless 0} v_{r} \epsilon^{m} f_{(m)} d \boldsymbol{v} \quad \text { for } v_{r} \lessgtr 0 \quad \text { at } r= \pm \frac{1}{2} \text {. }
$$

Then $f_{(0)}, f_{(1)}, f_{(2)}, f_{(3)}$ are defined so that each term in (2.31) cancels, that is,

$$
\begin{aligned}
& \text { order } \epsilon^{0}: \mathcal{A}^{0} f_{(0)}=Q\left(f_{(0)}\right) \\
& \text { order } \epsilon^{1}:\left(\mathcal{A}^{0}-D Q\left(f_{(0)}\right)\right) f_{(1)}=-\mathcal{A}^{1} f_{(0)} \\
& \text { order } \epsilon^{2}:\left(\mathcal{A}^{0}-D Q\left(f_{(0)}\right)\right) f_{(2)}= \frac{1}{2} D^{2} Q\left(f_{(0)}\right)\left(f_{(1)}, f_{(1)}\right)-\mathcal{A}^{2} f_{(0)}-\mathcal{A}^{1} f_{(1)}, \\
& \text { order } \epsilon^{3}:\left(\mathcal{A}^{0}-D Q\left(f_{(0)}\right)\right) f_{(3)}= D^{2} Q\left(f_{(0)}\right)\left(f_{(1)}, f_{(2)}\right) \\
&+\frac{1}{6} D^{3} Q\left(f_{(0)}\right)\left(f_{(1)}, f_{(1)}, f_{(1)}\right) \\
&-\mathcal{A}^{2} f_{(1)}-\mathcal{A}^{1} f_{(2)},
\end{aligned}
$$

with the boundary condition for $m=0,1,2,3$

$$
f_{(m)}= \pm \frac{2}{\pi T_{w}^{2}} \exp \left(-\frac{\boldsymbol{v}^{2}}{T_{w}}\right) \int_{v_{r} \gtrless 0} v_{r} f_{(m)} d \boldsymbol{v} \text { for } v_{r} \lessgtr 0 \quad \text { at } r= \pm \frac{1}{2} .
$$

The solving of problems (2.32)-(2.33) is the subject of the next two subsections, while the theorem is proved in sections 2.2.3 and 2.2.4.

2.2.1. Leading order $\boldsymbol{\epsilon}^{\mathbf{0}}$. Here we have to find $f_{(0)}$ that solves problem (2.32) with boundary condition (2.36). This problem is rewritten here for clarity:

$$
\begin{gathered}
v_{r} \partial_{r} f_{(0)}+\kappa(1-\kappa r)^{-1} v_{r} v_{s} \partial_{v_{s}} f_{(0)}-\kappa(1-\kappa r)^{-1} v_{s}^{2} \partial_{v_{r}} f_{(0)} \\
=\frac{1}{K_{0}} \rho_{(0)}\left(M\left[\rho_{(0)}, \boldsymbol{u}_{(0)}, T_{(0)}\right]-f_{(0)}\right),
\end{gathered}
$$

with boundary condition

$$
f_{(0)}= \pm \frac{2}{\pi T_{w}^{2}} \exp \left(-\frac{\boldsymbol{v}^{2}}{T_{w}}\right) \int_{v_{r} \gtrless 0} v_{r} f_{(0)} d \boldsymbol{v} \quad \text { for } v_{r} \lessgtr 0 \quad \text { at } r= \pm \frac{1}{2} .
$$

We have the following lemma.

Lemma 2.4. Any function of the form $f=\rho M\left[1,0, T_{w}(s)\right]$ where $\rho$ is a function of $s$ and $t$ only is a solution of problem (2.37)-(2.38).

Proof. This can be seen either by coming back to original variables $(\boldsymbol{x}, \boldsymbol{v})$ in (2.37) or by a direct computation. Indeed, since $f$ is a Maxwellian, the right-hand side of (2.37) vanishes. Moreover, since $f$ is independent of $r$, the first term of the left-hand side vanishes too. Then an easy computation shows that the velocity derivatives in the left-hand side cancel out (this is a direct consequence of the isotropy of $f$ w.r.t. $\boldsymbol{v}$ ). Finally, it is clear that $f$ satisfies boundary condition (2.38).

In fact it can be proved that these solutions to problem (2.37)-(2.38) are the only ones. This can easily be proved by using the entropy inequality, the Darrozès-Guiraud inequality (see [9] for details about this inequality), and the conservation laws. Then we state the following theorem (we shall not specify any regularity assumption or any function space because our aim here is only a formal theory).

Copyright (c) by SIAM. Unauthorized reproduction of this article is prohibited. 
TheOREm 2.5. Problem (2.37)-(2.38) admits a one-dimensional manifold of solutions given by $\left\{f=\rho(s, t) M\left[1,0, T_{w}(s)\right], \rho(s, t) \in \mathbb{R}^{+}\right\}$.

Consequently, $f_{(0)}$ is necessarily given by

$$
f_{(0)}=\rho_{(0)}(s, t) M\left[1,0, T_{w}(s)\right],
$$

where the density $\rho_{(0)}$ is still to be determined. Note that the corresponding "mass flux" is necessarily zero:

$$
j_{(0)}:=\int_{-1 / 2}^{1 / 2} \int_{\mathbb{R}^{3}} v_{s} f_{(0)} d \boldsymbol{v} d r=0 .
$$

As explained at the beginning of section 2.2, this zero mass flux condition justifies a posteriori the use of a large (parabolic) time scale. Moreover, note that the other macroscopic quantities of $f_{(0)}$ are obviously

$$
u_{s(0)}=u_{r(0)}=0, \quad T_{(0)}=T_{w}(s) .
$$

Therefore, from (2.25), we see that the flow velocity is of the first order in $\epsilon$. Consequently, the scale of typical flow velocity is $\epsilon\left(2 R T_{0}\right)^{1 / 2}$. Since the length scale we are interested in is $L_{s}$, the time scale given in (2.12) can be interpreted as

$$
t_{0}=\frac{L_{s}}{\epsilon\left(2 R T_{0}\right)^{1 / 2}}=\frac{\text { length of our interest }}{\text { typical flow velocity }} .
$$

2.2.2. First order $\epsilon^{1}$. Here we want to find a solution $f_{(1)}$ of $(2.33)$ with boundary condition (2.36). We can prove the existence of $f_{(1)}$ with the following lemma (note that in the statement that follows, $s$ and $t$ are simple parameters which are kept "frozen" and are simply omitted).

LEMmA 2.6. (i) Let $g=g(r, v)$ defined on $\left[-\frac{1}{2}, \frac{1}{2}\right] \times \mathbb{R}^{3}$ be given and $L$ be the linear operator $\left(\mathcal{A}^{0}-D Q\left(f_{(0)}\right)\right)$. Then the equation

$$
L f=g,
$$

with boundary condition (2.36), is solvable if and only if $g$ satisfies the solvability condition

$$
\langle g\rangle=0, \quad\langle g\rangle:=\int_{-1 / 2}^{1 / 2} \int_{\mathbb{R}^{3}} g(1-\kappa r) d v d r .
$$

Two such solutions differ by a term proportional to $M\left[1,0, T_{w}\right]$. A unique solution can be singled out if one requires that $\langle f\rangle=0$. This unique solution will formally be denoted by $f=L^{-1} g$ (and $L^{-1}$ is the "pseudoinverse" of $L$ ).

(ii) If $g$ is even or odd w.r.t. $v_{s}$, then $f=L^{-1} g$ has the same parity.

Proof. We again stress the fact that the parameter $s$ in Lemma 2.6 is frozen and that, consequently, $\kappa$ is just a constant parameter. For point (i), going back to the change of variables $(\boldsymbol{x}, \boldsymbol{v}) \rightarrow\left(s, r, v_{s}, v_{r}, v_{3}\right)$ according to (2.1), (2.2), and (2.5), we note that for $f$ and $\kappa$ independent of $s$, we have

$$
\boldsymbol{v} \cdot \nabla_{\boldsymbol{x}} f=v_{r} \partial_{r} f+\kappa(1-\kappa r)^{-1} v_{r} v_{s} \partial_{v_{s}} f-\kappa(1-\kappa r)^{-1} v_{s}^{2} \partial_{v_{r}} f .
$$

Therefore, back to the variables $(\boldsymbol{x}, \boldsymbol{v})$, problem (2.42) with boundary condition (2.36) is written

$$
\boldsymbol{v} \cdot \nabla_{\boldsymbol{x}} f-D Q\left(f_{0}\right)(f)=g, \quad \boldsymbol{x} \in \Omega^{\prime} \times \mathbb{R},
$$


where now $\Omega^{\prime}$ is the domain comprised between two disks: $\Omega^{\prime}=\left\{x \in \mathbb{R}^{2}, \kappa^{-1}-\frac{1}{2} \leq\right.$ $\left.|x| \leq \kappa^{-1}+\frac{1}{2}\right\}$. By adapting the theory of [7] and [13], the results follow easily. We note that the factor $(1-\kappa r)$ in the definition of $\langle f\rangle$ is just the Jacobian of the change of variables from $(\boldsymbol{x}, \boldsymbol{v})$ to $\left(s, r, v_{s}, v_{r}, v_{3}\right)$.

Point (ii) is a consequence of the fact that the linear operator $L$ preserves the parity w.r.t. $v_{s}$ : this is directly seen for $\mathcal{A}^{0}$ from $(2.27)$, and for $D Q\left(f_{(0)}\right)$ this is the result of a simple computation. Then $f$ is decomposed into its odd and even parts, and it is not difficult by using point (i) of this lemma to see that the odd (or even) part of $f$ is zero if $g$ is even (or odd, respectively).

Consequently, the existence of $f_{(1)}$ is guaranteed if the right-hand side of (2.33) satisfies the solvability condition (2.43). From (2.39) and (2.29), it is clear that $-\mathcal{A}^{1} f_{(0)}$ is odd w.r.t. $v_{s}$ and hence satisfies (2.43). Then we can denote by $f_{(1)}=$ $-L^{-1} f_{(0)}$ the unique solution of (2.33) with boundary condition (2.36).

Now we derive a more explicit expression for $f_{(1)}$ in order to obtain the value of $j_{(1)}$ in (2.22). We also obtain expressions for the transport coefficients $M_{P}$ and $M_{T}$ that are important for the numerical simulations.

Problem (2.33) can be written as

$$
\begin{gathered}
v_{r} \partial_{r} f_{(1)}+\kappa(1-\kappa r)^{-1} v_{r} v_{s} \partial_{v_{s}} f_{(1)}-\kappa(1-\kappa r)^{-1} v_{s}^{2} \partial_{v_{r}} f_{(1)} \\
=\frac{1}{K_{0}} \rho_{(0)}\left(M_{(1)}-f_{(1)}\right)-(1-\kappa r)^{-1} v_{s} \partial_{s} f_{(0)}, \\
M_{(1)}=f_{(0)}\left[\frac{\rho_{(1)}}{\rho_{(0)}}+\frac{2 u_{s(1)} v_{s}+2 u_{r(1)} v_{r}}{T_{w}}+\frac{T_{(1)}}{T_{w}}\left(\frac{\boldsymbol{v}^{2}}{T_{w}}-\frac{3}{2}\right)\right], \\
f_{(1)}= \pm \frac{2}{\pi T_{w}^{2}} \exp \left(-\frac{\boldsymbol{v}^{2}}{T_{w}}\right) \int_{v_{r} \gtrless 0} v_{r} f_{(1)} d \boldsymbol{v} \text { for } v_{r} \lessgtr 0 \quad \text { at } r= \pm \frac{1}{2} .
\end{gathered}
$$

From point (ii) of Lemma $2.6, f_{(1)}$ has the same parity (w.r.t. $v_{s}$ ) as $\mathcal{A}^{1} f_{(0)}$, which is odd. Consequently, $\rho_{(1)}, u_{r(1)}$ and $T_{(1)}$ are necessarily zero (see (A.5)-(A.8)). The second term of the right-hand side of (2.44) is an inhomogeneous term because $f_{(0)}$ is known at this point (see (2.39)). The explicit form of this inhomogeneous term reads

$$
-(1-\kappa r)^{-1} v_{s} \partial_{s} f_{(0)}=-(1-\kappa r)^{-1} v_{s} f_{(0)}\left[\partial_{s} \ln p_{(0)}+\left(\frac{\boldsymbol{v}^{2}}{T_{w}}-\frac{5}{2}\right) \partial_{s} \ln T_{w}\right],
$$

where $p_{(0)}=\rho_{(0)} T_{w}$.

In order to simplify the study of this problem (as well as its numerical approximation), we introduce the following variables:

$$
\phi(s, r, \boldsymbol{\zeta}, t):=\frac{f_{(1)}}{f_{(0)}}, \quad \boldsymbol{\zeta}:=\frac{\boldsymbol{v}}{\sqrt{T_{w}}} .
$$

The boundary value problem for $\phi$ relevant to problem (2.44)-(2.46) is

$\zeta_{r} \partial_{r} \phi+\kappa(1-\kappa r)^{-1} \zeta_{r} \zeta_{s} \partial_{\zeta_{s}} \phi-\kappa(1-\kappa r)^{-1} \zeta_{s}^{2} \partial_{\zeta_{r}} \phi$

$$
\begin{aligned}
& =\frac{1}{K}\left(M_{\phi}-\phi\right)-(1-\kappa r)^{-1} \zeta_{s}\left[\partial_{s} \ln p_{(0)}+\left(\frac{\zeta^{2}}{T_{w}}-\frac{5}{2}\right) \partial_{s} \ln T_{w}\right], \\
M_{\phi} & =2 \nu \zeta_{s}, \\
\phi & = \pm 2 \sqrt{\pi} \int_{\zeta_{r} \gtrless 0} \zeta_{r} \phi E d \zeta \quad \text { for } \zeta_{r} \lessgtr 0 \quad \text { at } r= \pm \frac{1}{2},
\end{aligned}
$$

Copyright $@$ by SIAM. Unauthorized reproduction of this article is prohibited. 
where

$$
\nu=\frac{u_{s(1)}}{\sqrt{T_{w}}}=\int \zeta_{s} \phi E d \zeta
$$

and

$$
E=\frac{1}{\pi^{3 / 2}} \exp \left(-\zeta^{2}\right)
$$

The variable $K$ in (2.48) is a kind of local Knudsen number defined by

$$
K=\frac{\sqrt{T_{w}}}{\rho_{(0)}} K_{0}=\frac{\sqrt{T_{w}}}{\rho_{(0)}} \frac{\left(2 R T_{0}\right)^{1 / 2}}{D A_{c} \rho_{0}}=\frac{\left(2 R T_{w}^{\text {dim }}\right)^{1 / 2}}{D A_{c} \rho_{(0)}^{\text {dim }}},
$$

where we wrote by $\rho_{(0)}^{\text {dim }}=\rho_{0} \rho_{(0)}$ and $T_{w}^{d i m}=T_{0} T_{w}$ the dimensional variables (we recall that $T_{0}$ and $\rho_{0}$ are reference values). Since $\rho_{(0)}$ depends on $s$ and $t$ and $T_{w}$ depends on $s, K$ also depends on $s$ and $t$.

In view of linearity of the problem and dependence of the inhomogeneous term on $\boldsymbol{\zeta}$, we can split $\phi$ into the following two (odd) parts:

$$
\phi(s, r, \boldsymbol{\zeta}, t)=\phi_{P}(r, \boldsymbol{\zeta} ; K(s, t), \kappa(s)) \partial_{s} \ln p_{(0)}+\phi_{T}(r, \boldsymbol{\zeta} ; K(s, t), \kappa(s)) \partial_{s} \ln T_{w}
$$

The boundary value problems for $\phi_{P}$ and $\phi_{T}$ can be expressed by introducing an index $J$ representing $P$ or $T$ as

$$
\begin{aligned}
& (2.55) \zeta_{r} \partial_{r} \phi_{J}+\kappa(1-\kappa r)^{-1} \zeta_{r} \zeta_{s} \partial_{\zeta_{s}} \phi_{J}-\kappa(1-\kappa r)^{-1} \zeta_{s}^{2} \partial_{\zeta_{r}} \phi_{J}=\frac{1}{K}\left(M_{\phi_{J}}-\phi_{J}\right)-I_{J}, \\
& (2.56) I_{P}=(1-\kappa r)^{-1} \zeta_{s}, \quad I_{T}=(1-\kappa r)^{-1} \zeta_{s}\left(\zeta^{2}-\frac{5}{2}\right), \\
& (2.57) M_{\phi_{J}}=2 \nu_{J} \zeta_{s},
\end{aligned}
$$

with boundary conditions

$$
\phi_{J}= \pm 2 \sqrt{\pi} \int_{\zeta_{r} \gtrless 0} \zeta_{r} \phi_{J} E d \zeta \quad \text { for } \zeta_{r} \lessgtr 0 \quad \text { at } r= \pm \frac{1}{2},
$$

and with

$$
\nu_{J}=\int \zeta_{s} \phi_{J} E d \zeta
$$

By solving these problems numerically, the first order solution $f_{(1)}$ can be obtained:

$$
f_{(1)}=f_{(0)}\left(\phi_{P} \partial_{s} \ln p_{(0)}+\phi_{T} \partial_{s} \ln T_{w}\right) \text {. }
$$

The corresponding "mass flux" of $f_{(1)}$ then is

$j_{(1)}:=\int_{-1 / 2}^{1 / 2} \int v_{s} f_{(1)} d \boldsymbol{v} d r=\int_{-1 / 2}^{1 / 2} \rho_{(0)} \sqrt{T_{w}}\left(\nu_{P} \partial_{s} \ln p_{(0)}+\nu_{T} \partial_{s} \ln T_{w}\right) d r$

$$
=\rho_{(0)} \sqrt{T_{w}}\left(M_{P} \partial_{s} \ln p_{(0)}+M_{T} \partial_{s} \ln T_{w}\right)=\sqrt{T_{w}} M_{P} \partial_{s} \rho_{(0)}+\frac{\rho_{(0)}}{\sqrt{T_{w}}}\left(M_{P}+M_{T}\right) \partial_{s} T_{w}
$$

Copyright (C) by SIAM. Unauthorized reproduction of this article is prohibited. 
where

$$
M_{P}(K, \kappa)=\int_{-1 / 2}^{1 / 2} \nu_{P} d r, \quad M_{T}(K, \kappa)=\int_{-1 / 2}^{1 / 2} \nu_{T} d r .
$$

Through their dependence on $\nu_{J}$ and hence on $\phi_{J}$, they depend only on the two parameters of problem (2.55)-(2.58) that are the local Knudsen number $K$ and the curvature $\kappa$.

We give a short comment on auxiliary problems (2.55)-(2.58). Physically, (2.55)(2.58) with $J=P$ describe a circulating flow between two coaxial circular cylinders driven by a constant (small) pressure gradient in the circumferential direction, and those with $J=T$ describe a circulating flow in the same geometry driven by a constant (small) gradient of the surface temperature of each cylinder in the circumferential direction. Indeed, if the BGK equation is written in cylindrical coordinates for a flow between two cylinders, and if we assume that, at first order, $f$ is such that the pressure gradient is small and constant, then after linearization, we exactly get (2.55)-(2.58) with $J=P$ (and also for $J=T$ if instead we assume a small temperature gradient on the wall). Such flows are physically unrealistic because the pressure of the gas or the temperature of the cylinders becomes multivalued. However, (2.55)-(2.58) make sense mathematically, and $M_{P}$ and $M_{T}$ are interpreted as the mass-flow rates of the artificial Poiseuille flow and thermal transpiration induced in the circumferential direction between two coaxial circular cylinders. If we take the limit of $\kappa \rightarrow 0$ (zero curvature limit), problems (2.55)-(2.58) reduce to the problems of the Poiseuille flow and thermal transpiration between two parallel plates, which have been widely studied in the literature (see, e.g., [11, 25, 19, 24, 26, 36, 30, 37, 31, 12]).

2.2.3. Diffusion model satisfied at the limit $\epsilon=0$ and order of approximation (proof of points (i) and (iii) of Theorem 2.3). It is now sufficient to solve problems (2.34) and (2.35), that is, to compute the second and third order terms $f_{(2)}$ and $f_{(3)}$ of the Hilbert expansion. However, these functions are not necessary for the numerical simulation of the diffusion model. To simplify the reading of this paper, these formal computations are deferred to Appendix B.

Now we assume that $f_{(2)}$ and $f_{(3)}$ are known. From (2.31), it is clear that the third order Hilbert approximation $f_{\epsilon}=f_{(0)}+\epsilon f_{(1)}+\epsilon^{2} f_{(2)}+\epsilon^{3} f_{(3)}$ satisfies the same equation as $f$ up to $O\left(\epsilon^{2}\right)$ terms, namely

$$
\begin{aligned}
\partial_{t} f_{\epsilon}+(1-\kappa r)^{-1} v_{s} \partial_{s} f_{\epsilon}+ & \epsilon^{-1} v_{r} \partial_{r} f_{\epsilon}+\epsilon^{-1} \kappa(1-\kappa r)^{-1} v_{r} v_{s} \partial_{v_{s}} f_{\epsilon} \\
& -\epsilon^{-1} \kappa(1-\kappa r)^{-1} v_{s}^{2} \partial_{v_{r}} f_{\epsilon}=\epsilon^{-2} Q\left(f_{\epsilon}\right)+O\left(\epsilon^{2}\right) .
\end{aligned}
$$

Provided that the $O\left(\epsilon^{2}\right)$ terms satisfy suitable estimates and that adequate initial and boundary conditions are prescribed, then it is possible to (formally) prove that $f_{\epsilon}$ is an order $\epsilon^{2}$ approximation of $f: f=f_{\epsilon}+O\left(\epsilon^{2}\right)$, and hence $f$ converges to $f_{(0)}$ as $\epsilon$ goes to 0 .

Consequently, the density of $f$ in a section of the channel satisfies $\varrho=\rho_{(0)}+$ $\epsilon \rho_{(1)}+O\left(\epsilon^{2}\right)$. Since $\rho_{(1)}$ is zero by the odd parity of $f_{(1)}$ w.r.t. $v_{s}$ (see the remark after $(2.46))$, we have $\varrho=\rho_{(0)}+O\left(\epsilon^{2}\right)$.

In a similar way, from the second order approximation of $f$ by $f_{\epsilon}$, we find that 
the mass flux of $f$ satisfies

$$
\begin{aligned}
j & =\frac{1}{\epsilon} \int_{-1 / 2}^{1 / 2} \int_{\mathbb{R}^{3}} v_{s}\left(f_{(0)}+\epsilon f_{(1)}+\epsilon^{2} f_{(2)}+O\left(\epsilon^{3}\right)\right) d \boldsymbol{v} d r \\
& =\frac{1}{\epsilon} j_{(0)}+j_{(1)}+\epsilon j_{(2)}+O\left(\epsilon^{2}\right) .
\end{aligned}
$$

From Appendix B, a parity argument again shows that the second order mass flux $j_{(2)}$ is zero. Since the zeroth order flux $j_{(0)}$ is also zero, we simply obtain the second part of (iii).

Finally, for point (i), passing to the limit $\epsilon=0$ in the continuity equation (2.18) directly gives the limit diffusion model (2.21)-(2.22), where coefficients $M_{P}$ and $M_{T}$ are given in (2.62). Note that for numerically solving this equation, one just needs to obtain $M_{P}$ and $M_{T}$ numerically for various values of $K$ and $\kappa$ (see section 4.2).

2.2.4. Sign of the diffusion coefficient (proof of point (ii) of Theorem 2.3). We consider (2.55) with $J=P$. In order to make $M_{P}$ appear in the equation, we multiply it by $\phi_{P} E(1-\kappa r)$ and integrate in $\left[-\frac{1}{2}, \frac{1}{2}\right] \times \mathbb{R}^{3}$ w.r.t. $r$ and $\boldsymbol{\zeta}$. This gives

$$
\begin{aligned}
& \int_{-1 / 2}^{1 / 2} \int_{\mathbb{R}^{3}} \zeta_{r} \partial_{r}\left(\frac{\phi_{P}^{2}}{2}\right) E(1-\kappa r) d \boldsymbol{\zeta} d r \\
& +\int_{-1 / 2}^{1 / 2} \int_{\mathbb{R}^{3}} \kappa\left(\zeta_{r} \zeta_{s} \partial_{\zeta_{s}}\left(\frac{\phi_{P}^{2}}{2}\right)-\zeta_{s}^{2} \partial_{\zeta_{r}}\left(\frac{\phi_{P}^{2}}{2}\right)\right) E d \boldsymbol{\zeta} d r \\
& \quad=\int_{-1 / 2}^{1 / 2} \int_{\mathbb{R}^{3}} \frac{1}{K}\left(2 \nu_{P} \zeta_{s} \phi_{P} E-\phi_{P}^{2} E\right)(1-\kappa r) d \boldsymbol{\zeta} d r-M_{P} .
\end{aligned}
$$

Now the idea is that the left-hand side is the flux across the boundaries of a section of the channel of "particles" represented by the (positive) distribution $\phi_{P}^{2} E$. Since boundary condition (2.58) and the odd parity of $\phi_{P}$ w.r.t. $\zeta_{s}$ imply that

$$
\phi_{P}=0 \quad \text { for } \zeta_{r} \lessgtr 0 \quad \text { at } r= \pm \frac{1}{2},
$$

this means that the flux of these particles is nonnegative. This can be proved explicitly as follows. First, a simple integration by parts of the first term of the left-hand side of (2.63) gives

$\int_{-1 / 2}^{1 / 2} \int_{\mathbb{R}^{3}} \zeta_{r} \partial_{r}\left(\frac{\phi_{P}^{2}}{2}\right) E(1-\kappa r) d \boldsymbol{\zeta} d r$

$=\left.\int_{\mathbb{R}^{3}} \zeta_{r}^{+}(1-\kappa r) \frac{\phi_{P}^{2}}{2}\right|_{r=\frac{1}{2}} E d \boldsymbol{\zeta}-\left.\int_{\mathbb{R}^{3}} \zeta_{r}^{-}(1-\kappa r) \frac{\phi_{P}^{2}}{2}\right|_{r=-\frac{1}{2}} E d \boldsymbol{\zeta}+\int_{-1 / 2}^{1 / 2} \int_{\mathbb{R}^{3}} \kappa \zeta_{r} \frac{\phi_{P}^{2}}{2} E d \boldsymbol{\zeta} d r$,

where $\zeta_{r}^{ \pm}$stand for the positive and negative parts of $\zeta_{r}$. Then for the second term of the left-hand side of (2.63), we note that the isotropy of $E$ w.r.t. $\zeta$ implies $\zeta_{r} \zeta_{s} \partial_{\zeta_{s}} E-\zeta_{s}^{2} \partial_{\zeta_{r}} E=0$. Therefore, $E$ can be put inside the derivatives, and an 
integration by parts gives

$$
\begin{aligned}
& \int_{-1 / 2}^{1 / 2} \int_{\mathbb{R}^{3}} \kappa\left(\zeta_{r} \zeta_{s} \partial_{\zeta_{s}}\left(\frac{\phi_{P}^{2}}{2}\right)-\zeta_{s}^{2} \partial_{\zeta_{r}}\left(\frac{\phi_{P}^{2}}{2}\right)\right) E d \boldsymbol{\zeta} d r \\
& =\int_{-1 / 2}^{1 / 2} \int_{\mathbb{R}^{3}} \kappa\left(\partial_{\zeta_{s}}\left(\zeta_{r} \zeta_{s} \frac{\phi_{P}^{2}}{2} E\right)-\zeta_{r} \frac{\phi_{P}^{2}}{2} E-\partial_{\zeta_{r}}\left(\zeta_{s}^{2} \frac{\phi_{P}^{2}}{2} E\right)\right) d \boldsymbol{\zeta} d r \\
& =-\int_{-1 / 2}^{1 / 2} \int_{\mathbb{R}^{3}} \kappa \zeta_{r} \frac{\phi_{P}^{2}}{2} E d \boldsymbol{\zeta} d r .
\end{aligned}
$$

Consequently, we add up the results of (2.64) and (2.65) to obtain that the left-hand side of $(2.63)$ is

$$
\begin{aligned}
& \int_{-1 / 2}^{1 / 2} \int_{\mathbb{R}^{3}} \zeta_{r} \partial_{r}\left(\frac{\phi_{P}^{2}}{2}\right) E(1-\kappa r) d \boldsymbol{\zeta} d r \\
& +\int_{-1 / 2}^{1 / 2} \int_{\mathbb{R}^{3}} \kappa\left(\zeta_{r} \zeta_{s} \partial_{\zeta_{s}}\left(\frac{\phi_{P}^{2}}{2}\right)-\zeta_{s}^{2} \partial_{\zeta_{r}}\left(\frac{\phi_{P}^{2}}{2}\right)\right) E d \boldsymbol{\zeta} d r \\
& =\left.\int_{\mathbb{R}^{3}} \zeta_{r}^{+}(1-\kappa r) \frac{\phi_{P}^{2}}{2}\right|_{r=\frac{1}{2}} E d \boldsymbol{\zeta}-\left.\int_{\mathbb{R}^{3}} \zeta_{r}^{-}(1-\kappa r) \frac{\phi_{P}^{2}}{2}\right|_{r=-\frac{1}{2}} E d \boldsymbol{\zeta},
\end{aligned}
$$

which is nonnegative (we remind the reader that by assumption $1-\kappa r>0$; see the beginning of section 2.1).

Finally, it remains to prove that the first term of the right-hand side of (2.63) is negative to conclude. The fact that it is nonpositive is expected since this property is a consequence of the fact that the linear operator $\frac{1}{f_{(0)}} D Q\left(f_{(0)}\right)$ is nonpositive selfadjoint in space $L^{2}\left(\mathbb{R}^{3} d v\right)$. However, we directly prove this assertion as follows. We remark that the integrand of the first term of the right-hand side of (2.63) can be rewritten as follows:

$$
2 \nu_{P} \zeta_{s} \phi_{P} E-\phi_{P}^{2} E=-\left(2 \nu_{P} \zeta_{s}-\phi_{P}\right)^{2} E+4 \nu_{P}^{2} \zeta_{s}^{2} E-2 \nu_{P} \zeta_{s} \phi_{P} E .
$$

Using this relation and the fact that $\int_{\mathbb{R}^{3}} \zeta_{s}^{2} E d \boldsymbol{\zeta}=\frac{1}{2}$, we find that the first term of the right-hand side of (2.63) turns into

$$
\begin{aligned}
& \int_{-1 / 2}^{1 / 2} \frac{1-\kappa r}{K}\left[-\int_{\mathbb{R}^{3}}\left(2 \nu_{P} \zeta_{s}-\phi_{P}\right)^{2} E d \boldsymbol{\zeta}+2 \nu_{P}^{2}-2 \nu_{P}^{2}\right] d r \\
& =-\frac{1}{K} \int_{-1 / 2}^{1 / 2} \int_{\mathbb{R}^{3}}(1-\kappa r)\left(2 \nu_{P} \zeta_{s}-\phi_{P}\right)^{2} E d \boldsymbol{\zeta} d r
\end{aligned}
$$

which is nonpositive. It can even be seen that this term is in fact negative: indeed, if not, then $\phi_{P}=2 \nu_{P} \zeta_{s}$ by (2.67). If we insert this into (2.55), we find an ordinary differential equation for $\nu_{P}$ that must be satisfied for every $\zeta_{r}$, which is not possible.

Consequently, relations (2.66) and (2.67) imply that $-M_{P}$ is positive, which proves point (ii) of Theorem 2.3 and concludes the proof of this theorem.

3. Diffusion model for discontinuous curvature. On the basis of the result in the previous section, we discuss the junction where the curvature changes discontinuously; see Figure 2. Let the position at the junction be $s=0$. We suppose that the temperature of the wall is continuous at the junction, i.e., $\left.T_{w}\right|_{s=0-}=\left.T_{w}\right|_{s=0+}$ (but the derivative of $T_{w}$ can be discontinuous there). 


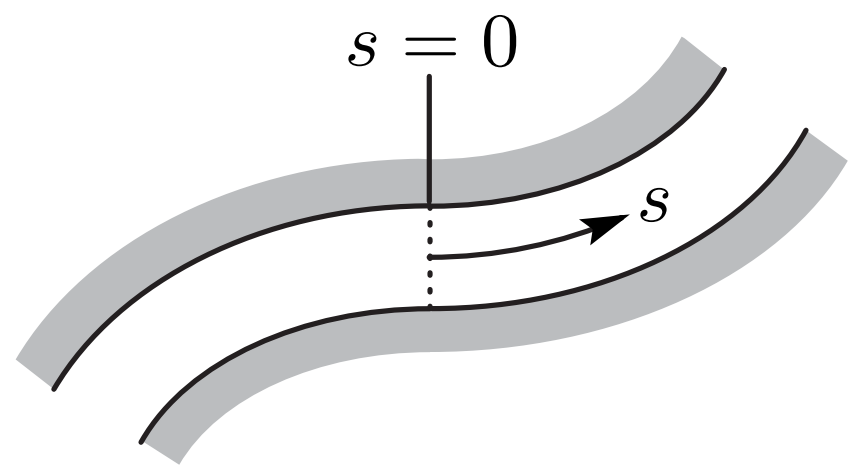

FIG. 2. Junction with discontinuous curvature.

The condition at the junction at the kinetic level is

$$
\left.f\right|_{s=0-}=\left.f\right|_{s=0+} \cdot
$$

Then it is easily seen that the fluxes at both sides are equal:

$$
\left.\int_{-1 / 2}^{1 / 2} \int_{\mathbb{R}^{3}} v_{s} f\right|_{s=0+} d \boldsymbol{v} d r=\left.\int_{-1 / 2}^{1 / 2} \int_{\mathbb{R}^{3}} v_{s} f\right|_{s=0-} d \boldsymbol{v} d r .
$$

Now the diffusion model (2.21)-(2.22) must be replaced by a transmission problem of the two following diffusion equations:

$$
\begin{aligned}
& \partial_{t} \rho_{(0)}^{\alpha}+\partial_{s} j_{(1)}^{\alpha}=0, \\
& j_{(1)}^{\alpha}=\sqrt{T_{w}} M_{P} \partial_{s} \rho_{(0)}^{\alpha}+\frac{\rho_{(0)}^{\alpha}}{\sqrt{T_{w}}}\left(M_{P}+M_{T}\right) \partial_{s} T_{w},
\end{aligned}
$$

where the superscript $\alpha$ is $L$ for the one-sided problem with $s \in]-\infty, 0[$ and $R$ for the one with $s \in] 0,+\infty[$. This problem requires two transmission conditions that are given in the following theorem.

THEOREM 3.1. With the junction conditions

$$
\left.\rho_{(0)}^{R}\right|_{s=0+}=\left.\rho_{(0)}^{L}\right|_{s=0-},\left.\quad j_{(1)}^{R}\right|_{s=0+}=\left.j_{(1)}^{L}\right|_{s=0-},
$$

the solution $\left(\rho_{(0)}^{\alpha}, j_{(1)}^{\alpha}\right)$ of $(3.3)-(3.4)$ is only a first order approximation of the density and mass flux $(\rho, j)$ of $f$.

A junction condition that preserves the second order approximation (away from $s=0)$ is

$$
\left.\rho_{(0)}^{R}\right|_{s=0+}=\left.(1+\epsilon d) \rho_{(0)}^{L}\right|_{s=0-},\left.\quad j_{(1)}^{R}\right|_{s=0+}=\left.j_{(1)}^{L}\right|_{s=0-},
$$

where $d$ is defined through an auxiliary linear kinetic problem which is given in the proof of the theorem.

3.1. Proof of Theorem 3.1. For clarity, here and in what follows, ()$^{ \pm}$put on functions of $s$ stands for their values at $s=0_{+}$and $s=0_{-}$, respectively. For one-sided functions with superscript $R$ or $L$, this superscript will be omitted when no confusion is possible. For instance $\left.\rho_{(0)}^{R}\right|_{s=0+}$ is denoted by $\rho_{(0)}^{+}$and $\left.\rho_{(0)}^{L}\right|_{s=0-}$ by $\rho_{(0)}^{-}$. 
For the junction condition (3.5), we define the Hilbert approximation $f_{\epsilon}^{\alpha}$ of $f$ as in section 2.2.3, where $f_{(0)}^{\alpha}$ is defined by $(2.39)$ with $\rho_{(0)}=\rho_{(0)}^{\alpha}$ in left or right subdomains. In the same way, the higher order terms are defined as in the previous sections. Then according to (3.5), $f_{\epsilon}^{\alpha}$ can satisfy the continuity relation (3.1) at $s=0$ only up to first order. Indeed, despite the fact that $f_{(0)}^{\alpha}$ satisfies (3.1) due to (3.5), this is not possible for $f_{(1)}^{\alpha}$, because the curvature has a discontinuity at $s=0$ and thus $\phi_{P}^{+} \neq \phi_{P}^{-}$and $\phi_{T}^{+} \neq \phi_{T}^{-}$. Therefore, the same arguments as in section 2.2 .3 give that $\rho_{(0)}^{\alpha}$ and $j_{(1)}^{\alpha}$ are only first order approximations.

The junction condition (3.6) is then constructed so as to maintain a second order approximation of $f$ at $s=0$, that is, to ensure that $f_{\epsilon}^{\alpha}$ satisfies (3.1) up to second order. Here we simply follow the derivation of [1]. In our case, the derivation is simpler since we do not have reflections between the two sides of the junction, but the varying curvature makes the computations a bit complicated. Classically, we introduce a pair of correctors, say $g^{\alpha}$, superimposed onto the Hilbert approximation $f_{\epsilon}$, which is needed only in a small region of size $\epsilon$ around $s=0$ :

$$
g^{\alpha}(y, r, \boldsymbol{v}, t) \rightarrow 0 \quad \text { as } y \rightarrow \pm \infty, \quad \alpha=R, L,
$$

where $y=s / \epsilon$. Here and in what follows, when the symbols \pm and $\alpha$ are present in the same expression, this must be understood as + and $R$ or - and $L$. Then $f_{\epsilon}$ is now defined by $f_{\epsilon}^{L}$ if $s<0$ and $f_{\epsilon}^{R}$ if $s>0$ as

$$
f_{\epsilon}^{\alpha}=f_{(0)}^{\alpha}+\epsilon\left(f_{(1)}^{\alpha}+g^{\alpha}(s / \epsilon)\right)+O\left(\epsilon^{2}\right), \quad \alpha=R, L .
$$

Note that as opposed to the classical Hilbert expansion, $f_{(0)}^{\alpha}$ here is allowed to depend on $\epsilon$ (as can be seen in the first relation of (3.6)). Inserting this expansion into (2.13) and (2.17) and separating the different order of $\epsilon$, we find that $f_{(k)}^{\alpha}$ are given by the same relations as in sections 2.2.1, 2.2.2, and Appendix B for $k=0,1,2,3$ and $\alpha=L, R$. Moreover, we also find a relation satisfied by $g^{\alpha}$ up to the order $O(\epsilon)$ in which we expand around $y=0$ all the coefficients that depend on $s=\epsilon y$. This gives the following equations:

$$
\begin{gathered}
\left(1-\kappa^{ \pm} r\right)^{-1} v_{s} \partial_{y} g^{\alpha}+v_{r} \partial_{r} g^{\alpha}+\kappa^{ \pm}\left(1-\kappa^{ \pm} r\right)^{-1} v_{r} v_{s} \partial_{v_{s}} g^{\alpha} \\
-\kappa^{ \pm}\left(1-\kappa^{ \pm} r\right)^{-1} v_{s}^{2} \partial_{v_{r}} g^{\alpha}=\frac{1}{K_{0}} \rho_{(0)}^{ \pm}\left(\bar{M}^{\alpha}-g^{\alpha}\right), \\
g^{\alpha}=\left\{\begin{array}{ccc}
+\frac{2}{\pi T_{w}^{2}} \exp \left(-\frac{\boldsymbol{v}^{2}}{T_{w}}\right) \int_{v_{r}>0} v_{r} g d \boldsymbol{v} & \text { for } v_{r}<0 & \text { at } r=+\frac{1}{2}, \\
-\frac{2}{\pi T_{w}^{2}} \exp \left(-\frac{\boldsymbol{v}^{2}}{T_{w}}\right) \int_{v_{r}<0} v_{r} g d \boldsymbol{v} & \text { for } v_{r}>0 & \text { at } r=-\frac{1}{2},
\end{array}\right.
\end{gathered}
$$

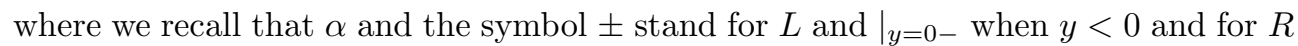
and $\left.\right|_{y=0+}$ when $y>0$. Moreover, $\bar{M}^{\alpha}$ is a linearized Maxwellian associated with $g^{\alpha}$ :

$$
\begin{aligned}
& \bar{M}^{\alpha}=f_{(0)}^{ \pm}\left[\frac{\bar{\rho}}{\rho_{(0)}^{ \pm}}+\frac{2 \bar{u}_{s} v_{s}+2 \bar{u}_{r} v_{r}}{T_{w}}+\frac{\bar{T}}{T_{w}}\left(\frac{\boldsymbol{v}^{2}}{T_{w}}-\frac{3}{2}\right)\right], \\
& \bar{\rho}=\int g^{\alpha} d \boldsymbol{v}, \quad \rho_{(0)}^{ \pm} \bar{u}_{s}=\int v_{s} g^{\alpha} d \boldsymbol{v}, \quad \rho_{(0)}^{ \pm} \bar{u}_{r}=\int v_{r} g^{\alpha} d \boldsymbol{v}, \\
& \frac{3}{2} \rho_{(0)}^{ \pm} \bar{T}=\int \boldsymbol{v}^{2} g^{\alpha} d \boldsymbol{v}-\frac{3}{2} \bar{\rho} T_{w} .
\end{aligned}
$$


Finally, note that in the expressions above, $T_{w}$ takes its value at $s=0$.

Now we want a solution $g^{\alpha}$ of (3.8)-(3.12) such that it vanishes for large $y$ (see (3.7)) and such that $f_{\epsilon}^{\alpha}$ can satisfy the continuity relation (3.1) up to second order. It is then sufficient to impose

$$
\left.\left(f_{(0)}^{R}+\epsilon\left(f_{(1)}^{R}+g^{R}\right)\right)\right|_{y=0+}=\left.\left(f_{(0)}^{L}+\epsilon\left(f_{(1)}^{L}+g^{L}\right)\right)\right|_{y=0-} .
$$

By using the form of $f_{(1)}^{\alpha}$ given by (2.60), it can be seen that this relation can be simplified by introducing the following new unknowns:

$$
\psi^{L}=\frac{g^{L}}{f_{(0)}^{-}} \quad \text { and } \quad \psi^{R}=\frac{g^{R}}{f_{(0)}^{-}}+\frac{\rho_{(0)}^{+}-\rho_{(0)}^{-}}{\epsilon \rho_{(0)}^{-}} .
$$

Then the boundary value problem for $g^{\alpha}$ can be written under the following equivalent problem for $\psi^{\alpha}$ :

$$
\begin{gathered}
\left(1-\kappa^{ \pm} r\right)^{-1} \zeta_{s} \partial_{y} \psi^{\alpha}+\zeta_{r} \partial_{r} \psi^{\alpha}+\kappa^{ \pm}\left(1-\kappa^{ \pm} r\right)^{-1} \zeta_{r} \zeta_{s} \partial_{\zeta_{s}} \psi^{\alpha} \\
-\kappa^{ \pm}\left(1-\kappa^{ \pm} r\right)^{-1} \zeta_{s}^{2} \partial_{\zeta_{r}} \psi^{\alpha}=\frac{1}{K^{ \pm}}\left(\bar{M}_{\psi^{\alpha}}-\psi^{\alpha}\right), \\
\psi^{\alpha}=\left\{\begin{array}{cc}
+\sqrt{\pi} \int_{\zeta_{r}>0} \zeta_{r} \psi^{\alpha} E d \zeta & \text { for } \zeta_{r}<0 \quad \text { at } r=+\frac{1}{2}, \\
-\sqrt{\pi} \int_{\zeta_{r}<0} \zeta_{r} \psi^{\alpha} E d \boldsymbol{\zeta} & \text { for } \zeta_{r}>0 \quad \text { at } r=-\frac{1}{2}, \\
\left.\psi^{L}\right|_{y=0-}=\left.\psi^{R}\right|_{y=0+}+\lambda(r, \boldsymbol{\zeta}),
\end{array}\right.
\end{gathered}
$$

where $K^{ \pm}=\left(\sqrt{T_{w}} / \rho_{(0)}^{ \pm}\right) K_{0}$ and

$$
\lambda=\sigma\left[\phi_{P}^{+}\left(\partial_{s} \ln p_{(0)}\right)^{+}+\phi_{T}^{+}\left(\partial_{s} \ln T_{w}\right)^{+}\right]-\phi_{P}^{-}\left(\partial_{s} \ln p_{(0)}\right)^{-}-\phi_{T}^{-}\left(\partial_{s} \ln T_{w}\right)^{-},
$$

with $\sigma=\rho_{(0)}^{+} / \rho_{(0)}^{-}$. The function $\bar{M}_{\psi^{\alpha}}$ is a linearized Maxwellian associated with $\psi^{\alpha}$ :

$$
\begin{aligned}
& \bar{M}_{\psi^{\alpha}}=\bar{\omega}+2 \bar{\nu}_{s} \zeta_{s}+2 \bar{\nu}_{r} \zeta_{r}+\bar{\tau}\left(\boldsymbol{\zeta}^{2}-\frac{3}{2}\right), \\
& \bar{\omega}=\int \psi^{\alpha} E d \boldsymbol{\zeta}, \quad \bar{\nu}_{s}=\int \zeta_{s} \psi^{\alpha} E d \boldsymbol{\zeta}, \quad \bar{\nu}_{r}=\int \zeta_{r} \psi^{\alpha} E d \boldsymbol{\zeta} \\
& \frac{3}{2} \bar{\tau}=\int\left(\boldsymbol{\zeta}^{2}-\frac{3}{2}\right) \psi E d \boldsymbol{\zeta} .
\end{aligned}
$$

Refer to (2.47) and (2.52), respectively, for the definitions of $\boldsymbol{\zeta}$ and $E$.

This problem is a double-sided Milne problem for which we assume that there exists a unique bounded solution. Moreover, we assume this solution has the following behavior for large $y$ :

$$
\begin{aligned}
& \psi^{L} \rightarrow 0 \quad \text { as } \quad y \rightarrow-\infty \\
& \psi^{R} \rightarrow d \quad \text { as } \quad y \rightarrow+\infty
\end{aligned}
$$

where $d$ is a constant. Since our goal is only a formal theory, we do not give a proof of the existence of $\psi^{\alpha}$. Some elements of the theory of double-sided Milne problems 
can be found in [15], where this kind of problem appeared for the first time. Some formal arguments related to our problem can be found in [1].

Consequently, we can find a pair of boundary layer correctors $\left(g^{L}, g^{R}\right)$ such that (3.13) is satisfied, but these correctors must also vanish for large $y$. From (3.14), (3.22), and (3.23), this can be satisfied only if

$$
\rho_{(0)}^{+}-\rho_{(0)}^{-}=d \times \epsilon \rho_{(0)}^{-},
$$

which is the first relation of the junction condition (3.6), and $d$ is the (computable) limit of $\psi^{R}$ for large $y$. The second relation of (3.6) is simply suggested by the continuity of $j$ in (3.2). Consequently, with these relations, the pair $\left(\rho_{(0)}^{L}, \rho_{(0)}^{R}\right)$ and the correctors $\left(g^{L}, g^{R}\right)$ allow us to define the Hilbert approximation $f_{\epsilon}^{\alpha}$ which is now by construction a second order approximation of $f$. The fact that $\left(\rho_{(0)}^{L}, \rho_{(0)}^{R}\right)$ and $\left(j_{(1)}^{L}, j_{(1)}^{R}\right)$ are second order approximations of $\rho$ and $j$ away from a tiny zone around $s=0$ follows from the same arguments as in section 2.2.3. This concludes the proof of the theorem.

Remark 3.1. The boundary value problem (3.15)-(3.23) is specified if the values of $K^{ \pm}, \kappa^{ \pm}$, and $\lambda$ are given: in fact, however, the linearity of the problem and the form of the inhomogeneous term $\lambda$ allow us to decrease the number of parameters by introducing the following:

$$
\begin{aligned}
& (3.24) \psi=\sigma\left[\psi_{P_{+}}\left(\partial_{s} \ln p_{(0)}\right)^{+}+\psi_{T_{+}}\left(\partial_{s} \ln T_{w}\right)^{+}\right]-\psi_{P_{-}}\left(\partial_{s} \ln p_{(0)}\right)^{-}-\psi_{T_{-}}\left(\partial_{s} \ln T_{w}\right)^{-}, \\
& (3.25) d=\sigma\left[d_{P_{+}}\left(\partial_{s} \ln p_{(0)}\right)^{+}+d_{T_{+}}\left(\partial_{s} \ln T_{w}\right)^{+}\right]-d_{P_{-}}\left(\partial_{s} \ln p_{(0)}\right)^{-}-d_{T_{-}}\left(\partial_{s} \ln T_{w}\right)^{-} .
\end{aligned}
$$

By substituting (3.24) and (3.25) into (3.15)-(3.23), we can split the problem into four problems for $\psi_{q}\left(q=P_{+}, T_{+}, P_{-}\right.$, and $\left.T_{-}\right)$; the explicit expressions are omitted here because they are straightforward. Then $d_{q}$ is a function of only four parameters $K^{ \pm}$ and $\kappa^{ \pm}$. Moreover, $d_{q}\left(K^{+}, K^{-}, \kappa^{ \pm}\right)-d_{q}\left(K^{-}, K^{-}, \kappa^{ \pm}\right)=O(\epsilon)$ because $\rho_{(0)}^{+}-\rho_{(0)}^{-}=$ $O(\epsilon)$ from (3.6) and thus $K^{+}-K^{-}=O(\epsilon)$. Accordingly, since $d$ is multiplied by $\epsilon$ in (3.6), the condition preserves the approximation up to second order in $\epsilon$ even if we replace $d_{q}\left(K^{+}, K^{-}, \kappa^{ \pm}\right)$by $d_{q}\left(K^{-}, K^{-}, \kappa^{ \pm}\right)$(or by $d_{q}\left(K^{+}, K^{+}, \kappa^{ \pm}\right)$). Therefore, in the actual application in the following, we regard $d_{q}$ as a function of three parameters $K, \kappa^{ \pm}$, i.e., $d_{q}=d_{q}\left(K, \kappa^{ \pm}\right)$.

Remark 3.2. If the double-sided Milne problem is rewritten in terms of $f_{\epsilon}^{\alpha}$, it is very similar to a shock structure problem. As a consequence, the width of the transition layer from the left to right state is of the order of $\epsilon$, as it is in a shock structure. However, there are some important differences between these two problems. For instance, the state at infinity in the double-sided Milne problem is $f_{0}+\epsilon f_{1}$ : it is not Maxwellian, and it is even not one- but two-dimensional ( $f_{1}$ depends on $s$ and $r$ ).

3.2. Approximation of $\boldsymbol{d}$. Since solving the two-dimensional double-sided Milne problem (3.15)-(3.23) is not an easy task, we shall use a formula for approximated value of $d$, following the idea of [1]. This idea already appeared in [14] and [15]; see also a review on this problem and further references in [17]. The formula relies on the following lemma and assumption.

Lemma 3.2. For problem (3.15)-(3.23), we have

$$
\int_{-1 / 2}^{1 / 2} \int \zeta_{s}\left(-\phi_{P}^{\alpha}(r,-\boldsymbol{\zeta} ; s=0 \pm)\right) \psi^{\alpha} E d \boldsymbol{\zeta} d r=C_{ \pm} \quad \text { for } y \gtrless 0,
$$

where $C_{-}$and $C_{+}$are constants. 
Assumption 3.1. For boundary value problem (3.15)-(3.23), $\psi^{\alpha}$ at $y=0$ is supposed to be such that

$$
\left.\psi\right|_{y=0 \pm} ^{\alpha}=b_{ \pm}, \quad b_{ \pm}= \pm\left. 2 \sqrt{\pi} \int_{-1 / 2}^{1 / 2} \int_{\zeta_{s} \gtrless 0} \zeta_{s} \psi^{\alpha}\right|_{y=0 \pm} E d \boldsymbol{\zeta} d r \quad \text { for } \zeta_{s} \lessgtr 0,
$$

where $b_{+}$and $b_{-}$are constants.

The proof of Lemma 3.2 is a consequence of the boundedness of $\psi^{\alpha}$. It can be found in a straightforward way by referring to Appendix B of [1] and is omitted here. Assumption 3.1 gives an approximation of the outgoing distributions $\psi^{\alpha}$ at the junction $s=0$ by equilibrium states, which is often sufficient in many kinetic boundary layer computations.

Now if we perform the integration in (3.27) by using (3.17), it turns out that $b_{+}=b_{-}:=b$. By computing $C_{-}$at $y=0_{-}$by using (3.17) with $\psi^{\alpha}=b$, we find

$$
C_{-}=b M_{P}^{-}-\int_{-1 / 2}^{1 / 2} \int_{\zeta_{s}<0} \zeta_{s} \lambda \phi_{P}\left(r,-\boldsymbol{\zeta} ; s=0_{-}\right) E d \boldsymbol{\zeta} d r .
$$

On the other hand, we have $C_{-}=0$ from the condition (3.22) at $y=-\infty$. Therefore,

$$
b=\left(M_{P}^{-}\right)^{-1} \int_{-1 / 2}^{1 / 2} \int_{\zeta_{s}<0} \zeta_{s} \lambda \phi_{P}\left(r,-\boldsymbol{\zeta} ; s=0_{-}\right) E d \boldsymbol{\zeta} d r
$$

Similarly, computations of $C_{+}$at $y=0_{+}$and $y=\infty$ give us

$$
d=b+\left(M_{P}^{+}\right)^{-1} \int_{-1 / 2}^{1 / 2} \int_{\zeta_{s}>0} \zeta_{s} \lambda \phi_{P}\left(r,-\zeta ; s=0_{+}\right) E d \boldsymbol{\zeta} d r .
$$

From (3.28) and (3.29), we obtain the formula for $d$ :

$\left.d=\int_{-1 / 2}^{1 / 2} \int_{\zeta_{s}>0} \zeta_{s} \lambda\left[\left(M_{P}^{+}\right)^{-1} \phi_{P}\left(r,-\boldsymbol{\zeta} ; s=0_{+}\right)-\left(M_{P}^{-}\right)^{-1} \phi_{P}\left(r,-\boldsymbol{\zeta} ; s=0_{-}\right)\right]\right] E d \boldsymbol{\zeta} d r$.

By inserting the explicit expression of $\lambda(3.18)$ and the form of (3.25) into this formula, we can also obtain the formulae for $d_{q}\left(q=P_{+}, T_{+}, P_{-}\right.$, and $\left.T_{-}\right)$.

4. Numerical approximation of the diffusion model. In this section we present the numerical approximation which will be used for the applications in the following section. First, we will show the discretization scheme for the diffusion model (2.21)-(2.22); then we will give a brief comment on how we calculate the numerical values of the diffusion coefficients $M_{P}$ and $M_{T}$.

4.1. Numerical scheme for the diffusion model. In the applications in the following section, we will consider the steady state, i.e., $\partial / \partial t \equiv 0$. In that case, the diffusion model reduces to

$$
\sqrt{T_{w}} M_{P} \partial_{s} \rho+\frac{\rho}{\sqrt{T_{w}}}\left(M_{P}+M_{T}\right) \partial_{s} T_{w}=j,
$$

where $j$ is a constant (even in the case of a discontinuous curvature, $j$ is constant throughout the entire channel due to the continuity of $j$ imposed by the second connection condition of (3.6)). In this section, the subscripts (0) attached to $\rho$ and (1) to $j$, which indicate the order of expansion, are dropped to avoid complexity. 
To obtain the numerical solution of (4.1), space variable $s$ is discretized with grid points $s_{(i)}=i \Delta s$ for $i=0,1, \ldots, i_{\max }$. Values of the functions on the grid point $s_{(i)}$ are indicated by the subscript $(i)$, e.g., $\rho_{(i)}=\rho\left(s_{(i)}\right)$ (which should not be confused with the subscript for the expansion in the previous sections). We employ an iterative process to overcome the nonlinearity of (4.1). That is, the numerical solution $\rho_{(i)}$ is obtained as the limit of the sequence $\rho_{(i)}^{(n)}(n=0,1, \ldots$,$) calculated by the following$ scheme:

$$
\sqrt{T_{w(i)}} M_{P(i)}^{(n-1)} D \rho_{(i)}^{(n)}+\frac{\rho_{(i)}^{(n)}}{\sqrt{T_{w(i)}}}\left(M_{P(i)}^{(n-1)}+M_{T(i)}^{(n-1)}\right)\left(\partial_{s} T_{w}\right)_{(i)}=j,
$$

where

$$
M_{P(i)}^{(n-1)}=M_{P}\left(\frac{\sqrt{T_{w(i)}}}{\rho_{(i)}^{(n-1)}} K_{0}, \kappa_{(i)}\right), \quad M_{T(i)}^{(n-1)}=M_{T}\left(\frac{\sqrt{T_{w(i)}}}{\rho_{(i)}^{(n-1)}} K_{0}, \kappa_{(i)}\right),
$$

and

$$
D \rho_{(i)}^{(n)}=\left\{\begin{array}{l}
\frac{\rho_{(i)}^{(n)}-\rho_{(i-1)}^{(n)}}{\Delta s} \text { or } \\
\frac{3 \rho_{(i)}^{(n)}-4 \rho_{(i-1)}^{(n)}+\rho_{(i-2)}^{(n)}}{2 \Delta s} .
\end{array}\right.
$$

The upper approximation is used for the points next to the boundary and the junction, and the lower is taken otherwise.

At junctions, we have two limiting values of physical quantities such as $\rho, \kappa$, and the derivative of $T_{w}$. We let $s=s_{(m)}$ be the curvilinear abscissae of the junction and denote by ()$_{(m)}^{ \pm}$the physical quantities at the limit $s=s_{(m) \pm}$. We adopt the following scheme for the junction:

$$
\rho_{(m)}^{+(n)}=\rho_{(m)}^{-(n)}\left(1+\epsilon d_{(m)}^{(n-1)}\right)
$$

where $d_{(m)}^{(n)}$ is computed in terms of the discretized version of (3.25):

$$
d_{(m)}^{(n)}=d_{P+(m)}^{(n)} \nabla p_{(m)}^{+(n)}+d_{T+(m)}^{(n)} \nabla T_{w(m)}^{+}-d_{P-(m)}^{(n)} \nabla p_{(m)}^{-(n)}-d_{T-(m)}^{(n)} \nabla T_{w(m)}^{-},
$$

with

$$
d_{q(m)}^{(n)}=d_{q}\left(\frac{\sqrt{T_{w(m)}}}{\rho_{(m)}^{-(n)}} K_{0}, \kappa_{(m)}^{ \pm}\right) \quad(q=P+, T+, P-, \text { and } T-),
$$

and

$$
\begin{array}{r}
\nabla T_{w(m)}^{ \pm}=\mp \frac{1}{T_{w(m)}} \frac{3 T_{w(m)}-4 T_{w(m \pm 1)}+T_{w(m \pm 2)}}{2 \Delta s}, \\
\nabla p_{(m)}^{ \pm(n)}=\nabla T_{w(m)}^{ \pm} \mp \frac{1}{\rho_{(m)}^{ \pm(n)}} \frac{3 \rho_{(m)}^{ \pm(n)}-4 \rho_{(m \pm 1)}^{(n)}+\rho_{(m \pm 2)}^{(n)}}{2 \Delta s} .
\end{array}
$$

Copyright (c) by SIAM. Unauthorized reproduction of this article is prohibited. 
If an analytical form of $T_{w}$ is given, $\nabla T_{w(m)}^{ \pm}$can be replaced by the analytical expression of $\left.\partial_{s} \ln T_{w}\right|_{s=s_{(m) \pm}}$. Relation (4.8) gives an approximation of $\partial_{s} \ln p=$ $\partial_{s} \ln T+\partial_{s} \ln \rho$. Note that (4.6) is the discretized expression of (3.25) in which $\sigma$ is replaced by unity: this does not affect the approximation (4.5) up to the order of $\epsilon$ because $\sigma=\frac{\rho^{+}}{\rho^{-}}=1+O(\epsilon)$. The solution process starts by giving some values to $\rho_{(i)}^{(0)}$ and $j$. When $\rho_{(i)}^{(n-1)}$ is known, $\rho_{(i)}^{(n)}$ is calculated by (4.2) with the boundary condition at $i=0$. The process is terminated when the results of two successive iterations agree within a specified tolerance, typically of order $10^{-10}$. We note that during this iteration process $j$ is never updated. The more detailed treatment of $j$ and the boundary condition is dependent on the physical situation. It will be mentioned in section 5 .

Incidentally, we have also tested an alternative scheme which excludes the iteration process. More precisely, we replaced $M_{P(i)}^{(n-1)}$ and $M_{T(i)}^{(n-1)}$ in (4.2) by the following $\bar{M}_{P(i-1)}^{(n)}$ and $\bar{M}_{T(i-1)}^{(n)}$ :

$$
\bar{M}_{P(i-1)}^{(n)}=M_{P}\left(\frac{\sqrt{T_{w(i)}}}{\rho_{(i-1)}^{(n)}} K_{0}, \kappa_{(i)}\right), \quad \bar{M}_{T(i-1)}^{(n)}=M_{T}\left(\frac{\sqrt{T_{w(i)}}}{\rho_{(i-1)}^{(n)}} K_{0}, \kappa_{(i)}\right) .
$$

Then $\rho_{(i)}^{(n)}$ can be calculated explicitly only with the boundary condition at $i=0$ (without iteration). We compared the results with the two different methods (iterative and noniterative methods) in detail. As a result, we found that the two methods converge to the same solution as the grid is refined. However, the iterative method converges faster and seems more accurate. Therefore, we employ the iterative method throughout this paper.

4.2. Numerical computation of the transport coefficients. When we implement the numerical computation of the diffusion model (4.1), the numerical data of the coefficients $M_{P}$ and $M_{T}$ are necessary. In the present work, we construct numerical databases of these coefficients as functions of $K$ and $\kappa$ (see the end of section 2.2.2) in the following way. First, we solve the auxiliary problem (2.55)-(2.58) numerically for many sets of $K$ and $\kappa$; the number of sets for each $M_{J}(J=P, T)$ is 732 in $0.02 \leq K \leq 1000$ and $0.0001 \leq \kappa \leq 1.9$ (the data for the special case of $\kappa=0$ are derived from $[36,37])$. We then interpolate these discrete data by means of the cubic spline method to make the continuous values of $M_{P}$ and $M_{T}$ available.

We employ a finite-difference method for the numerical analysis of the problem $(2.55)-(2.58)$. One technical difficulty in the finite-difference analysis is caused by the fact that the distribution function $\phi_{J}$ around $r=1 / 2$ has discontinuities in the velocity space because the boundary at $r=1 / 2$ is convex (the distribution function around convex bodies generally contains discontinuities [40]). To capture the discontinuities accurately, we adopt the technique developed by Sugimoto and Sone [43] for their study of strong evaporation from its cylindrical condensed phase. Since the whole procedure of the present finite-difference analysis is a straightforward application of that described in [43], we omit the detailed description of the scheme in the present paper and show only the results of the analysis: Figures 3 and 4 are the threedimensional plots of $-M_{P}\left(M_{P}\right.$ is nonpositive; see point (ii) of Theorem 2.3) and $M_{T}$ as functions of $K$ and $\kappa$.

Incidentally, we have also performed the asymptotic analyses for small and large Knudsen numbers, in order to complement the data for $K<0.02$ and $K>1000$, where the direct numerical analysis is not efficient. We omit the description of these analyses, 


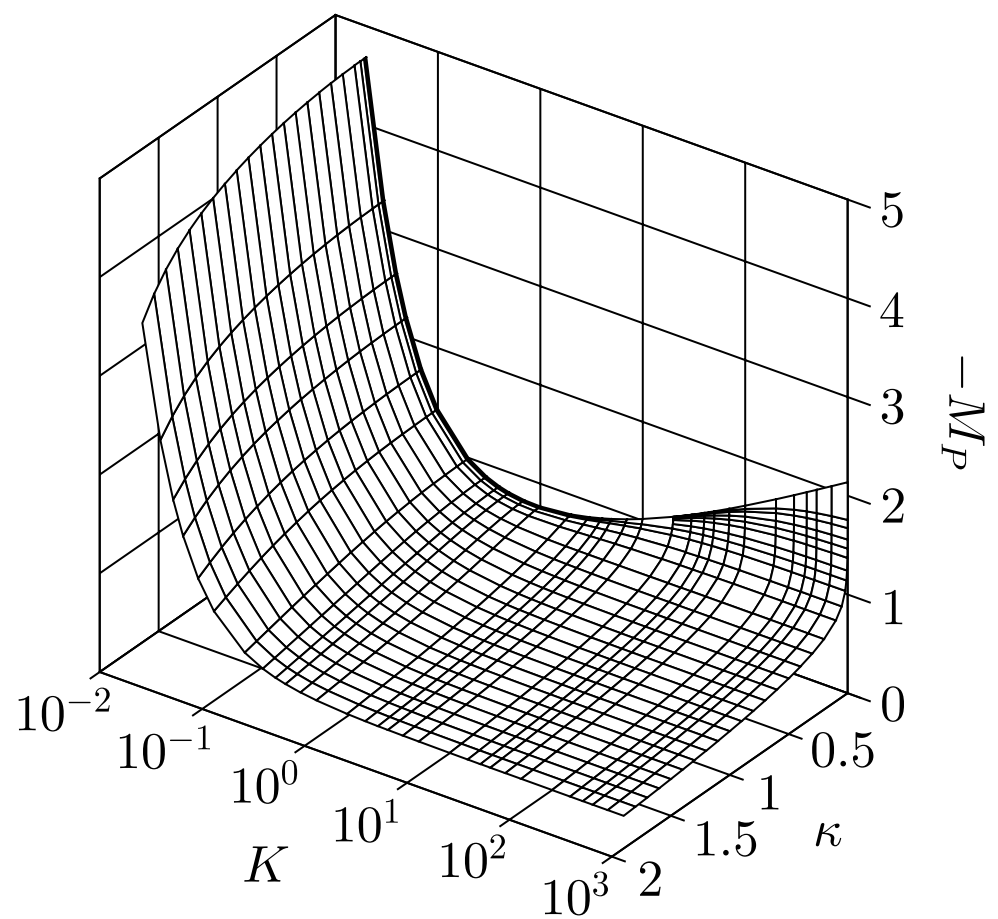

Fig. 3. Plot of $-M_{P}$ for $0.02 \leq K \leq 1000$ and $0 \leq \kappa \leq 1.9$. All the data are obtained by means of the present finite-difference analysis, except for those with $\kappa=0$, which are derived from [37].

however, because the numerical data obtained by the finite-difference analysis are sufficient for the application in the present paper.

If we use the connection condition at junctions up to the order of $\epsilon$ (see (3.6)), we also need a database for the jump coefficients $d_{q}$ with $q=P_{+}, T_{+}, P_{-}$, and $T_{-}$ (or $d$; see (3.25)). However, since $d_{q}$ is a function of three parameters $K$ and $\kappa^{ \pm}$(see Remark 3.1), it is not an easy task to construct a database of it for the whole range of parameters. Thus, in the present work, we pick up a few sets of $\kappa^{ \pm}$which are necessary for the applications in the following section and evaluate $d_{q}$ by means of the formula (3.30) (with (3.18) and (3.25)) for 30 values of $K$ in $0.02 \leq K \leq 1000$ for each set of $\kappa^{ \pm}$. By interpolating those values with the cubic spline method, we make the database of $d_{q}$ a continuous function of $K$.

5. Application: Simulation of two different devices. In this section, we apply the diffusion model and connection conditions to specific problems and show some numerical results. In section 5.1, we consider a ring-shaped channel, where a circulating flow is induced. To validate the present diffusion model, we compare the flux of the induced flows - as well as the macroscopic quantities - obtained by the diffusion model with those by the direct numerical analysis of the BGK equation (obtained by a method presented by the first three authors of this paper in [2]). Then, in section 5.2, we investigate the pumping effect of the circulating flow created in a cascade system made up of a number of basic units, each of which is part of the ring-shaped channel. We examine the effect of the parameters, such as the curvature, Knudsen number, and the number of units, on the performance of the pump. 


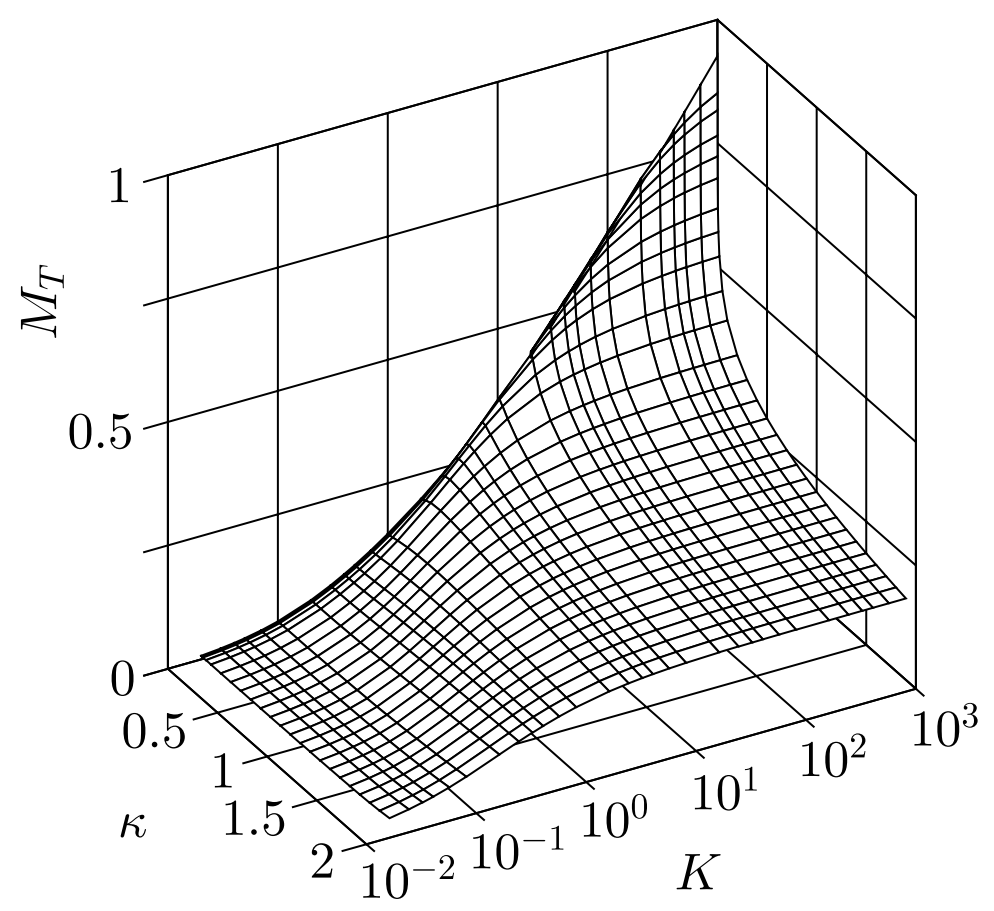

FIG. 4. Plot of $M_{T}$ for $0.02 \leq K \leq 1000$ and $0 \leq \kappa \leq 1.9$. See the caption of Figure 3 .

5.1. Circulating flow. We consider a gas in a (two-dimensional) ring-shaped channel with a uniform width, consisting of two straight channels and two semicircular channels. A schematic of the problem is shown in Figure 5 (top), where $s$ is the (dimensional) coordinate along the median line, $D$ is the width of the channel, $L$ is the length of the straight segments, and $R_{c}$ is the radius of the median line of the semicircular segments. The temperature distribution along the channel walls, which is a function of (dimensional) $s$ (see section 2.1), is shown in Figure 5 (bottom), where $T_{L}$ is the temperature of the walls at positions $\mathrm{A}$ and $\mathrm{C}$, and $T_{H}\left(>T_{L}\right)$ is that at positions $\mathrm{B}$ and $\mathrm{D}$. We investigate the steady behavior of the gas in the circuit on the basis of the diffusion model as well as the direct numerical analysis of the BGK equation.

To apply the diffusion model to this problem, we specify some notations used in section 2. We define the length scale along the median line of the channel as $L_{s}:=L+\pi R_{c}$, which is relevant because the temperature of the walls indeed varies with this length scale (note that the curvature is constant except at the positions A, $\mathrm{B}, \mathrm{C}$, and $\mathrm{D})$. We take $T_{L}$ and the average density over the whole ring $\rho_{a v}$ as the reference temperature $T_{0}$ and reference density $\rho_{0}$, respectively.

If we formulate the problem by means of the dimensionless variables in (2.10) and (2.11), we find that it is characterized by the four dimensionless parameters: Knudsen number $K_{0}\left[=\left(2 R T_{L}\right)^{1 / 2} /\left(D A_{c} \rho_{a v}\right)\right]$; (dimensionless) curvature of the semicircular segments $\kappa\left(=D / R_{c}\right)$; the ratio of the straight segments to the whole length of the median line $B\left(=L / L_{s}\right)$; and the temperature ratio $T_{H} / T_{L}$. It should be noted that the small parameter $\epsilon\left(=D / L_{s}\right)$ is not independent of the above parameters; a simple calculation shows that the following relation holds:

$$
\epsilon=\kappa(1-B) / \pi .
$$



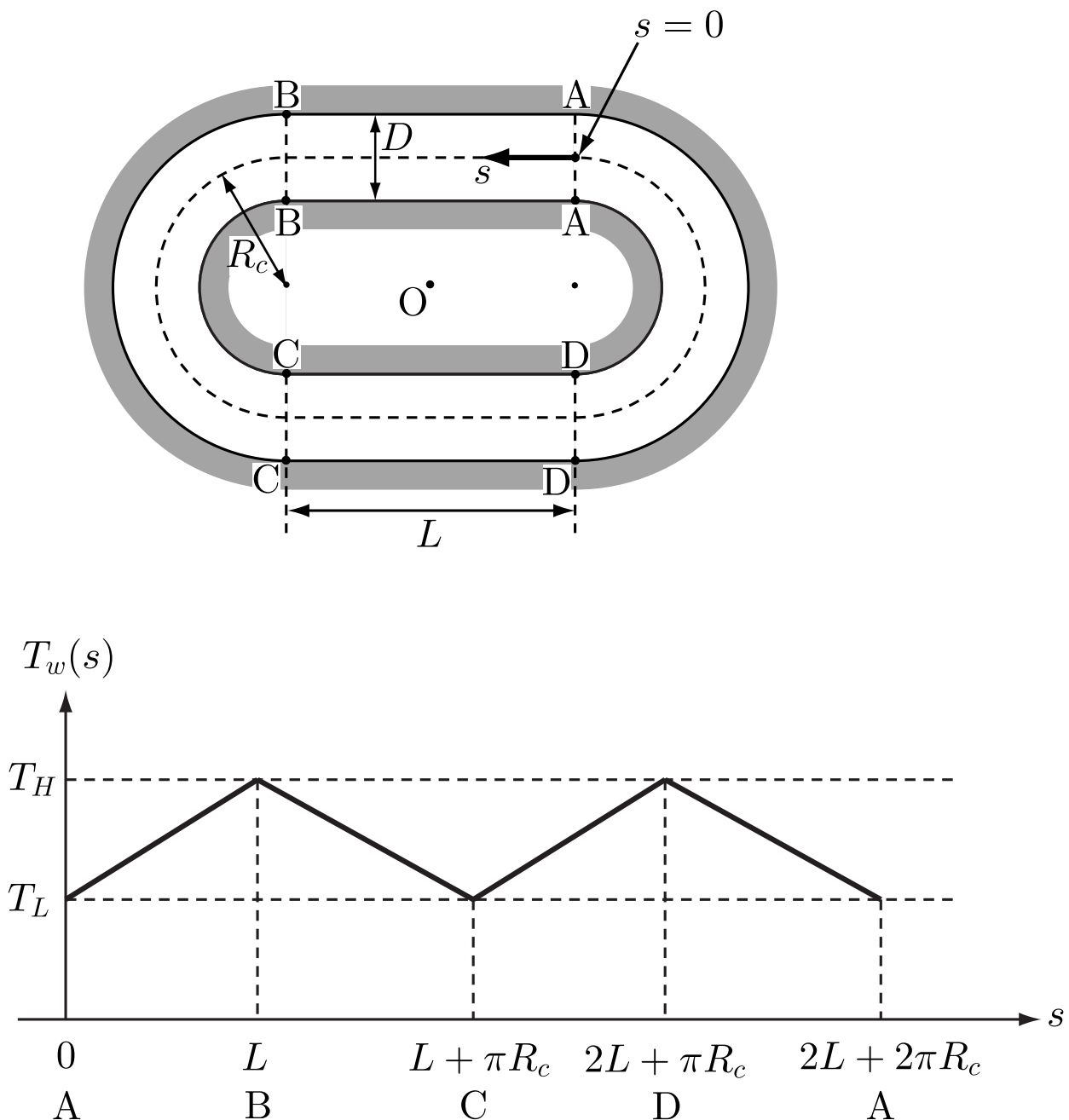

FIG. 5. Geometry (top) and temperature distribution (bottom) of the ring-shaped channel.

We briefly give some information supplemental to section 4.1 about the numerical scheme for the diffusion model. Because the geometry is symmetric w.r.t. the center $\mathrm{O}$ of the ring, we can restrict the computational space to $0 \leq s \leq 1$ (in what follows, $s$ indicates the dimensionless abscissa along the median line of the channel normalized by $L_{s}$ ) by imposing the periodic boundary condition at $s=0$ and $s=1$. We seek the solution to (4.1) that satisfies the periodic boundary condition in the following way. First, we choose an arbitrary value of $j$ and solve (4.1) numerically by means of the iterative method described in section 4.1 (here and in what follows in this section, subscripts (0) attached to $\rho$ and (1) attached to $j$ in the diffusion equation (2.21)(2.22) are dropped). The obtained solution generally does not satisfy the periodic condition, i.e., $\rho(s=0) \neq \rho(s=1)$. Thus we try again with a value of $j$ slightly different from the previous one; the new value of $j$ is sought so that the difference $\Delta \rho=|\rho(s=0)-\rho(s=1)|$ becomes smaller. We repeat this procedure until $\Delta \rho$ drops under a certain value, typically $10^{-10}$. In addition to the periodic condition, we have the normalizing condition for $\rho$, i.e., $\int_{0}^{1} \rho d s=1$. This condition is numerically 
enforced by the following method: for the first step of the iteration described in section 4.1 , we choose an arbitrary value of $\rho(s=0)$; then we multiply the obtained $\rho(0 \leq s \leq 1)$ by a constant so that the normalizing condition is satisfied. From the next step of the iteration, we use the value of $\rho(s=0)$ of the previous step.

In Figure 6, we show the distribution of the dimensionless density $\rho$, temperature $T$, and pressure $p(=\rho T)$ of the gas, averaged over the cross-section, for the following set of parameters: $K_{0}=0.5, B=0.5, T_{H} / T_{L}=3$, and $\kappa=1$ (a), 0.5 (b), and 0.2 (c). In the figure, the corresponding results of the direct numerical analysis of the BGK equation [2] are also shown for comparison. According to relation (5.1), the corresponding values of $\epsilon$ to $\kappa=1,0.5$, and 0.2 are $0.159,0.0796$, and 0.0318 , respectively. Therefore, the diffusion model, which is derived under the assumption $\epsilon \ll 1$, gives better approximation in Figure 6(c) than in Figure 6(a). In every case, however, the diffusion model shows sufficient agreement with the results of the direct numerical analysis.

Let us denote by $\mathcal{M}$ the dimensionless mass-flow rate of the gas across one section (per unit time and unit thickness in the $x_{3}$ direction):

$$
\mathcal{M}(s)=\int_{-1 / 2}^{1 / 2} \int_{\mathbb{R}^{3}} v_{s} f d \boldsymbol{v} d r .
$$

From (2.20), we see that $\mathcal{M}(s)=\epsilon j(s)$, which is approximated by $\epsilon j_{(1)}$ with the diffusion model. In the present problem, the circulating flow is induced in the clockwise direction. Since the coordinate $s$ is taken in the counterclockwise direction (Figure 5), the mass-flow rate $\mathcal{M}$ is negative. We show in Figure 7 the mass-flow rate obtained by the diffusion model for different Knudsen numbers and curvatures in the case of $B=0.5$ and $T_{H} / T_{L}=3$. More specifically, $-\mathcal{M}$ versus $K_{0}$ is plotted by $\square$ in the figure for $\kappa=1,0.5$, and 0.2 . When the curvature is large or the channel is thick $(\kappa=1)$, the mass-flow rate is large, and as the curvature decreases (or the channel becomes thinner), the mass-flow rate decreases. In the figure, we also plot the corresponding mass-flow rate obtained by the direct numerical analysis of the BGK equation [2]. The result of the diffusion model differs from that of the direct numerical analysis when $\kappa$ is large (or $\epsilon$ is large) as in Figure 6, but the two results approach each other asymptotically with the decrease of $\kappa$ (or $\epsilon$ ).

Although the mass-flow rate in the steady state is independent of $s$ theoretically, its numerical values show some fluctuations due to the numerical error in the direct numerical analysis. The position of the error bars shown in Figure 7 indicates the value averaged over $0 \leq s \leq 1$, and the length of the vertical error bar indicates the standard deviation from the average, which is an estimation of the numerical error.

5.2. Pumping effect. In this section, we consider a cascade system as shown in Figure 8 to investigate the pumping effect of the circulating flow examined in the previous section. The system consists of two kinds of basic units jointed alternately; one is the part $\mathrm{ABC}$ of Figure 5 (top), and the other is the mirror image of the part CDA with respect to the line CD. We let $s$ be the dimensionless coordinate normalized by $L_{s}$ along the median line of the channel, whose origin is located at the upper end in Figure 8. Accordingly, the lower end is located at $s=N$ when $N$ basic units are jointed.

We first consider the case where both ends of the channel are closed by diffusely reflecting walls kept at temperature $T_{L}$. In this case, a pressure difference at two ends is expected instead of the circulating flow. We compare the steady pressure distri- 


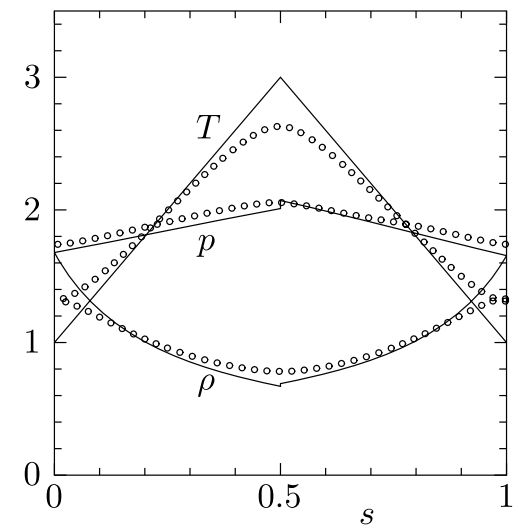

(a)

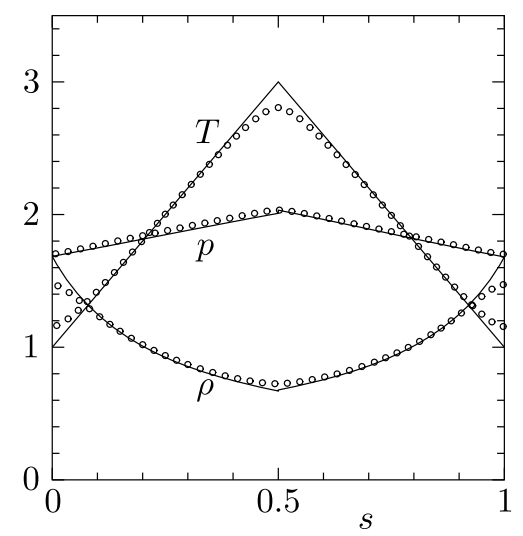

(b)

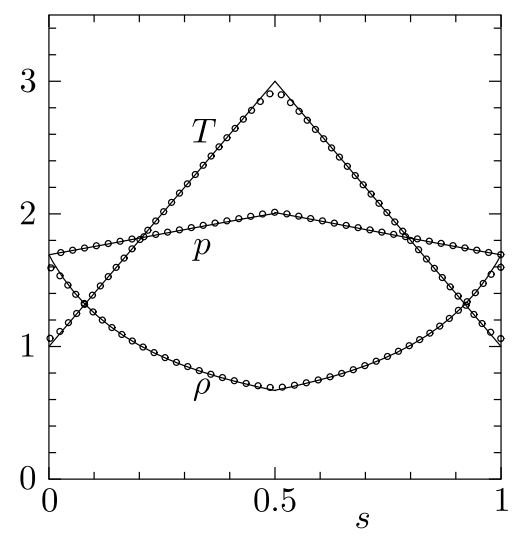

(c)

FIG. 6. The distribution of the dimensionless density $\rho$, temperature $T$, and pressure $p(=\rho T)$ of the gas, averaged over the cross-section, in the case of ring-shaped geometry. $K_{0}=0.5, B=0.5$, $T_{H} / T_{L}=3$, and $\kappa=1$ (a), $0.5(\mathrm{~b})$, and 0.2 (c). The solid line indicates the results by the diffusion model (2.21)-(2.22) with the connection condition (3.6), and the symbol $\bigcirc$ indicates the corresponding results by the direct numerical analysis of the BGK equation [2].

Copyright (c) by SIAM. Unauthorized reproduction of this article is prohibited. 


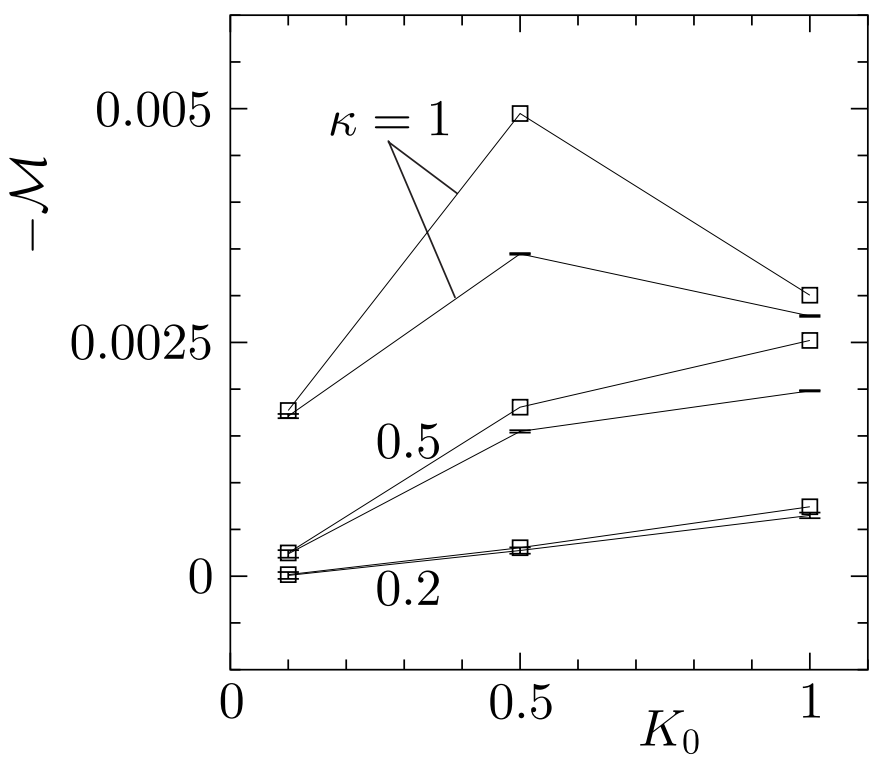

FIG. 7. The mass-flow rate $-\mathcal{M}$ versus the global Knudsen number $K_{0}$ for three different curvatures $\kappa=1,0.5$, and 0.2 , in the case of $B=0.5$ and $T_{H} / T_{L}=3 . \square$ indicates $-\mathcal{M}$ obtained by the diffusion model (2.21)-(2.22) with connection condition (3.6). The position of the error bar indicates the corresponding mass-flow rate obtained by the direct numerical analysis of the BGK equation [2], averaged along the channel; the length of the vertical error bar indicates the standard deviation from the average.

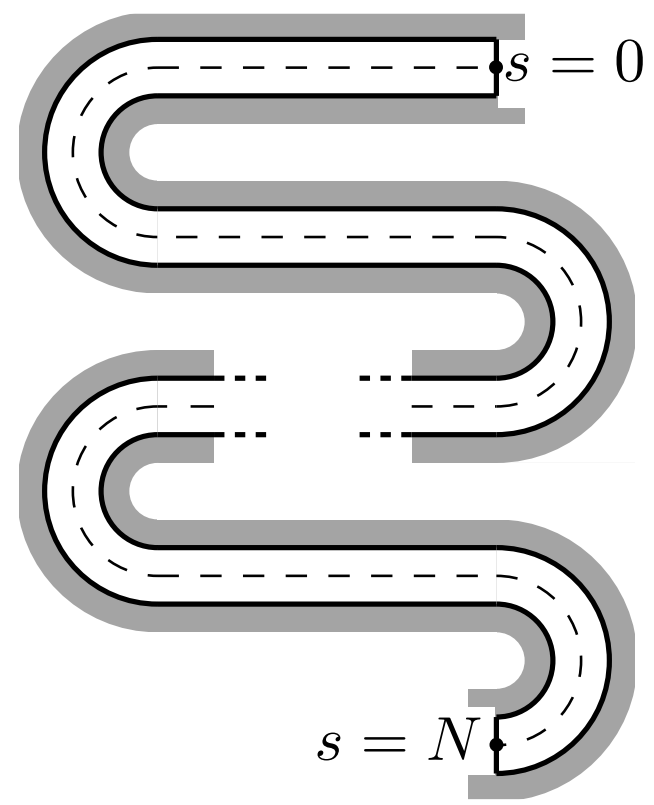

FIG. 8. Geometry of the cascade system.

Copyright (C) by SIAM. Unauthorized reproduction of this article is prohibited. 
bution obtained by the diffusion model with that obtained by the direct numerical analysis of the full BGK equation, in order to prove the validity of the diffusion model.

If we take the same quantities for the reference values as in the previous subsection and formulate the problem by means of the dimensionless variables given in (2.10) and (2.11), we also have the same dimensionless parameters that should be assigned to specify the physical situation: $K_{0}, \kappa, B$, and $T_{H} / T_{L}$, supplemented with $N$ in this problem.

We mention the boundary conditions that should be imposed in the diffusion model for the present problem. Since both ends are closed, we have $j=0$ at $s=0$ and $s=N$. From this condition, we can easily see that $j \equiv 0$ throughout the channel because we consider the steady state (see (4.1) and the sentence just after). In addition, we have the normalizing condition $\int_{0}^{N} \rho \mathrm{d} s=1$. The solution procedure for the diffusion model here is simpler than that described in the previous subsection, because now we know the value of $j(=0)$; it suffices to implement the iteration procedure as described in section 4.1 with making the normalizing condition satisfied in each iteration step.

In Figures 9 and 10 we show the steady pressure distribution along the channel for $\kappa=1$ and 0.2 , respectively, in the case of $K_{0}=0.5, B=0.5$, and $T_{H} / T_{L}=3$. The results for $N=1$ (a), 2 (b), 4 (c), and 8 (d) with $\kappa=1$ are shown in Figure 9, and the results for $N=1$ (a), 2 (b), and 4 (c) with $\kappa=0.2$ are shown in Figure 10. In each figure, the corresponding results by the direct numerical analysis of the BGK equation are also plotted by $\circ$ for comparison. The results show very good agreement in the case of $\kappa=0.2$. However, the discrepancy of two results is rather large in the case of $\kappa=1$. This is again because from relation (5.1) the larger $\kappa$ is, the larger $\epsilon$ is and accordingly the less accurate the approximation of the diffusion model is. From these results, it is seen that $\kappa$ must be small to obtain an accurate approximation by the diffusion model. We may expect, however, that the diffusion model does provide sufficient information, even for the case of $\kappa=1$, when we investigate the basic features of the device, such as dependence of the pressure difference on the physical parameters. Therefore, we proceed to further investigation of the pumping effect of the cascade system by means of the diffusion model.

From now on, we slightly change the problem for convenience in examining the pressure difference at two ends: we consider the case where the end at $s=N$ is closed by diffusely reflecting walls kept at temperature $T_{L}$, and the end at $s=0$ is open and kept at pressure $p_{e}$. Correspondingly, we choose $\rho_{e}=p_{e} /\left(R T_{L}\right)$ (i.e., the density at $s=0)$ as the reference density rather than the average density over the channel. In this case, we have the boundary condition $\rho=1$ at $s=0$, instead of the normalizing condition, and we can directly implement the iterative procedure described in section 4.1.

Some results based on the diffusion model for the pressure distribution along the channel are shown in Figures 11 and 12. Figure 11 shows the case of $N=1$ : (a) shows the dependence of the results on $K_{0}$; (b) on $\kappa$; (c) on $T_{H} / T_{L}$; and (d) on $B$. From Figure 11(a), we see that the pressure difference is the largest at some intermediate Knudsen number around $K_{0}=2$. Figure 11(b) and (c) tells us that the larger curvature provides larger pressure difference, and so does the larger temperature ratio. In contrast, the length ratio $B$ of the straight part and the semicircular part does not have a significant effect on the pressure difference (Figure 11(d)). Figure 12 shows the case of $N=100$ for three different values of the curvature $\kappa=1$ (a), 0.5 (b), and 0.2 (c). In each figure, the results of three different values of Knudsen number $K_{0}$ are shown. It seems, from the figure, that there exists an optimal value of $K_{0}$ for 


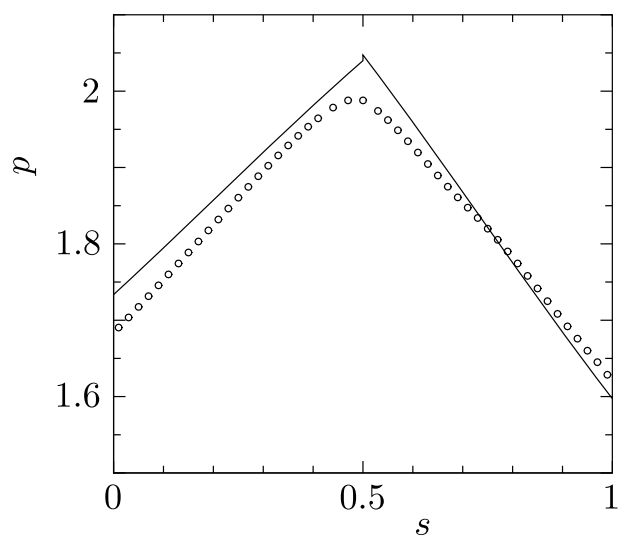

(a)

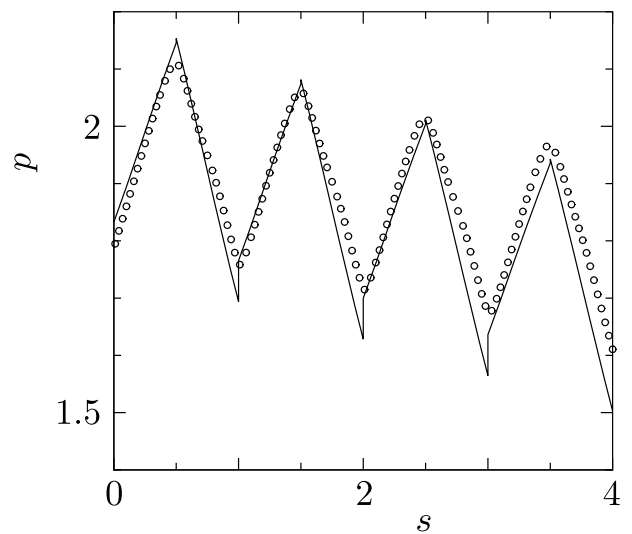

(c)

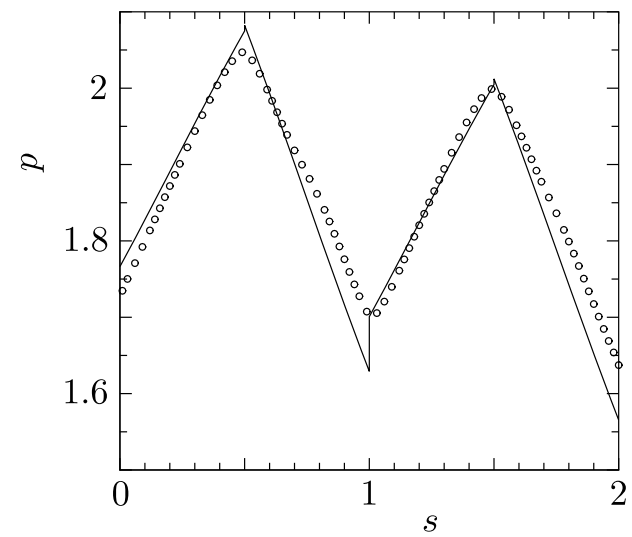

(b)

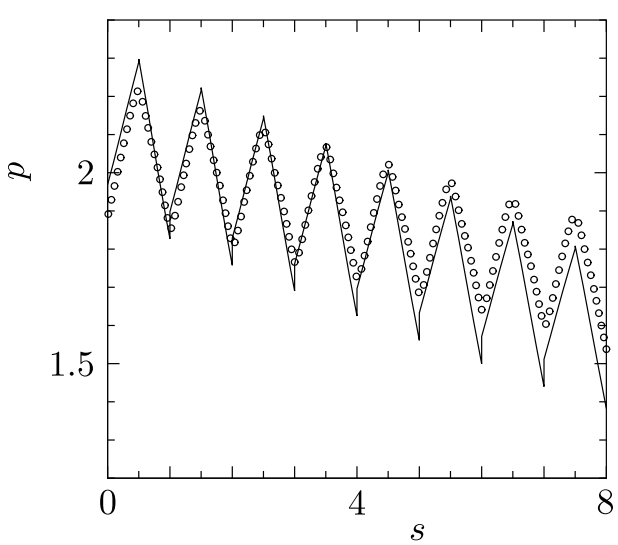

(d)

FIG. 9. Dimensionless pressure $p$ versus $s$ for $N=1$ (a), 2 (b), 4 (c), and 8 (d) in the case of $\kappa=1, K_{0}=0.5, B=0.5$, and $T_{H} / T_{L}=3$. The solid line indicates the result obtained by the diffusion model (2.21)-(2.22) with connection condition (3.6), and $\bigcirc$ indicates the results obtained by the direct numerical analysis of the BGK equation [2].

gaining a large pressure difference, and the value is dependent on the curvature.

In order to examine the dependence of the pressure difference on the Knudsen number in more detail, we have carried out the computations for many values of $K_{0}$, each with $N=100$, the results of which are shown in Figure 13. In this figure, following the idea of [41] (see also [5, 3]), $p(s+1) / p(s)$ and $K(s+1)$ are plotted in the vertical axis as functions of the local Knudsen number $K(s)$ (see (2.53)) for integer values of $s$. The curve is obtained as the union of several overlapping pieces of curves obtained for various values of $K_{0}$, each with $N=100$. Moreover, for each position $s$, since $p$ is discontinuous, the right value $p\left(s_{+}\right)$is chosen for calculations of $p(s+1) / p(s)$. From the figure, we see that there is a minimum value of the pressure ratio at a value of $K(\cdot)$, and the value $(\approx 0.97)$ is almost the same for different values of $\kappa$; in other words, the degree of pressure difference we can attain is almost the same for different $\kappa$ if we use some appropriate region of $K(\cdot)$, although, as seen from Figure 7, the flux of the circulating flow induced in the ring-shaped channel is smaller 


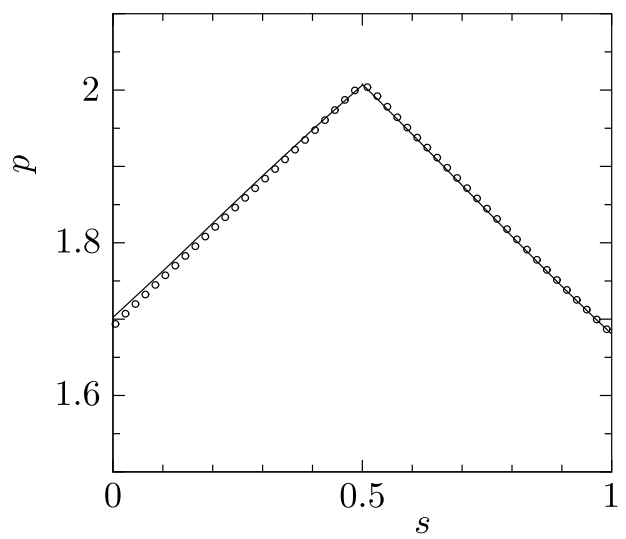

(a)

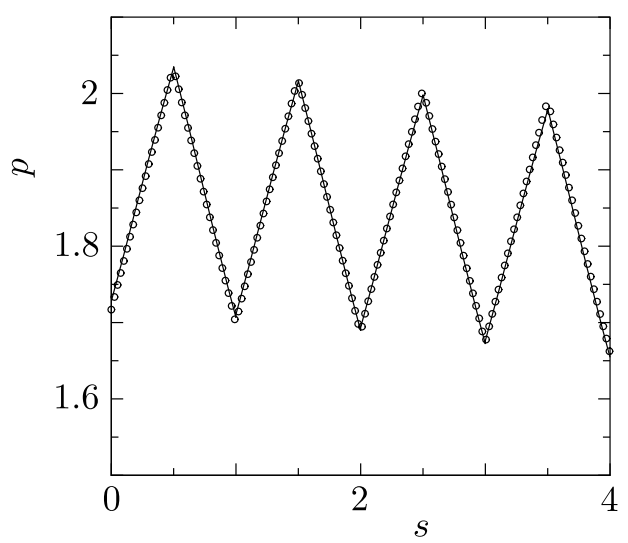

(c)

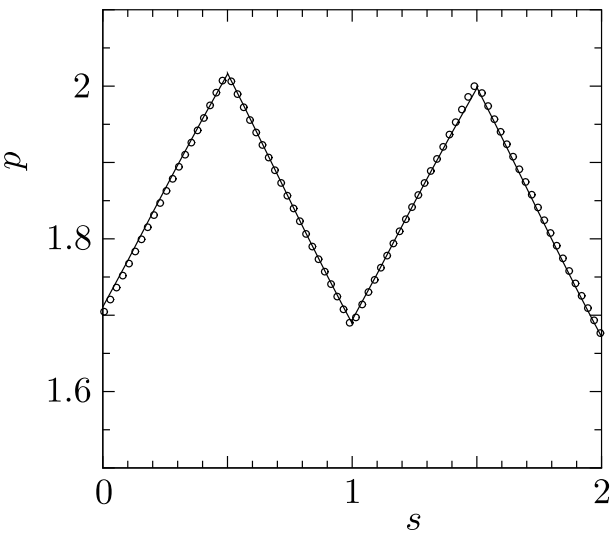

(b)

FIG. 10. Steady pressure distribution along the channel for $N=1$ (a), 2 (b), and 4 (c) in the case $\kappa=0.2, K_{0}=0.5, B=0.5$, and $T_{H} / T_{L}=3$. See the caption of Figure 9 .

for small $\kappa$ (see also [3]).

Even if the curves in Figure 13 have been obtained with a 100 unit device only, it is observed that the results obtained with a larger number of units lie on the same curves (see also [41]). Consequently, these curves can be used to estimate the pressure ratio when two reservoirs are connected by the cascade system composed of an arbitrarily large number (say $\mathcal{N}$ ) of the basic units in the following way (cf. [41, 5, 3]). Let $s=0$ be the entrance of the high-pressure side. If we know the local Knudsen number at the entrance $K(0)$, we can read the values of pairs $[p(1) / p(0), K(1)]$ from the figure. Then, with the aid of the $K(1)$, we can read the next pair $[p(2) / p(1), K(2)]$. By continuing this procedure until we know $p(\mathcal{N}) / p(\mathcal{N}-1)$, we can know the series of values $p(1) / p(0), p(2) / p(1), \ldots, p(\mathcal{N}) / p(\mathcal{N}-1)$, and then the pressure ratio of the two sides $p(\mathcal{N}) / p(0)$ can be obtained by simple multiplications of them.

6. Conclusion. We have presented a one-dimensional fluid model for a rarefied gas flow produced - as a result of the effect of thermal creep as well as that of pressure difference - in a two-dimensional curved channel. This model is a nonlin- 


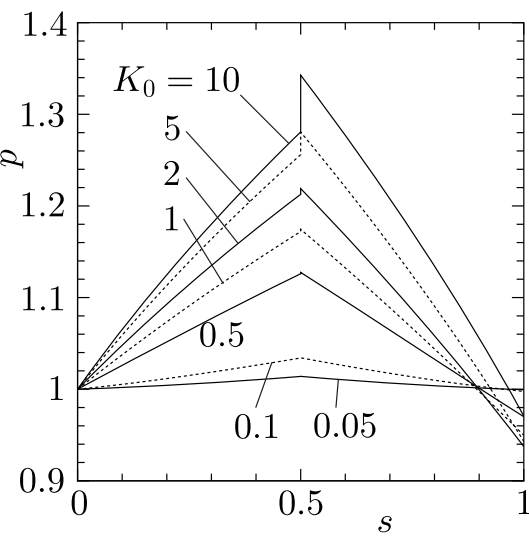

(a)

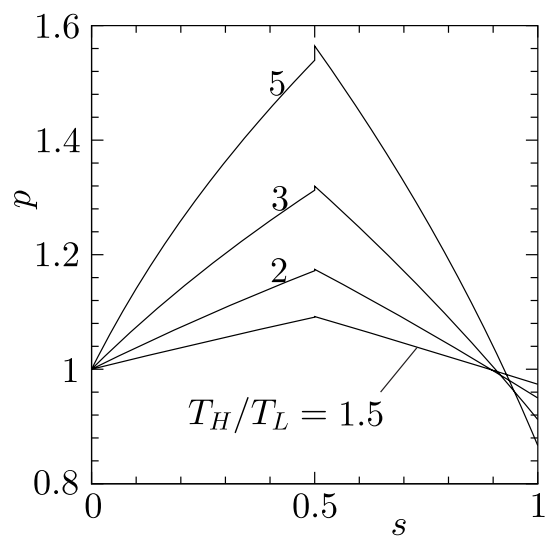

(c)

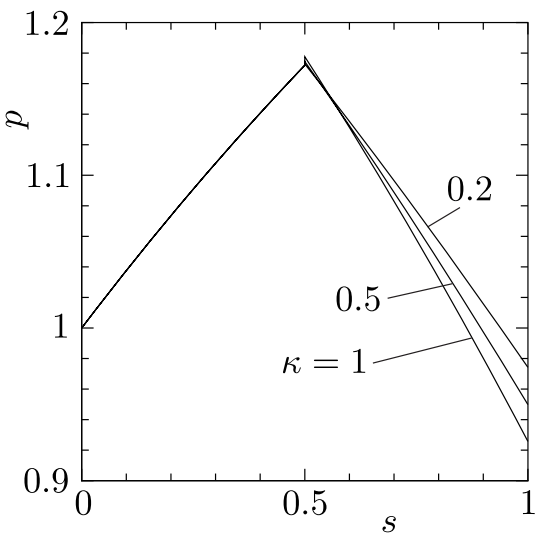

(b)

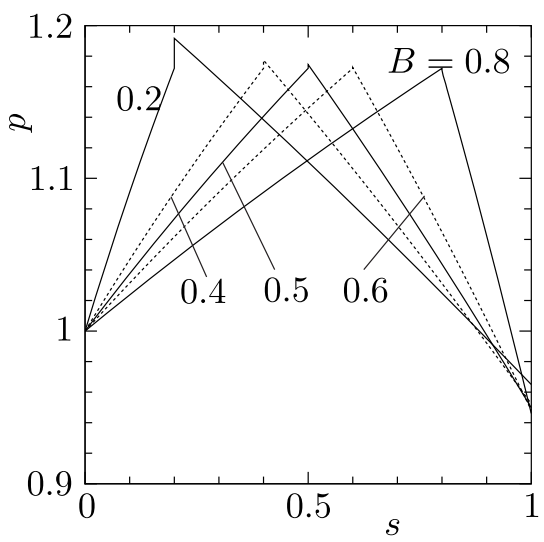

(d)

FIG. 11. Dimensionless pressure $p$ versus $s$ for $N=1$ in the case of (a) $\kappa=0.5, B=0.5$, $T_{H} / T_{L}=2$, and $K_{0}=0.05,0.1,0.5,1,2,5$, and 10 ; (b) $K_{0}=1, B=0.5, T_{H} / T_{L}=2$, and $\kappa=1,0.5$, and 0.2 ; (c) $\kappa=0.5, K_{0}=1, B=0.5$, and $T_{H} / T_{L}=1.5,2,3$, and 5 ; and $(\mathrm{d}) \kappa=0.5$, $K_{0}=1, T_{H} / T_{L}=2$, and $B=0.8,0.6,0.5,0.4$, and 0.2 . The dashed line used for some cases is just for legibility.

ear convection-diffusion equation that relies on a diffusion approximation of a kinetic model. The transport coefficients are numerically obtained from the geometry and the characteristics of the flow.

By using a numerical approximation of our fluid model, it has been shown that it compares very well with the original kinetic model. Moreover, it allows very fast simulation of a pumping device made of a very long cascade system, as opposed to full kinetic simulations. Consequently, we feel that this model may be very useful for further studies of this Knudsen compressor. In particular, since an intensive optimization study is necessary to determine the optimal parameters of our device so as to maximize the compression ratio, the numerical computation needed for such a study can be efficiently made with our model.

We also mention that this new device is the core of a large project in which we also have investigated efficient numerical solving of the full kinetic equation (see [2]) and also the applicability of other kinds of fluid approximations (see [23]). 


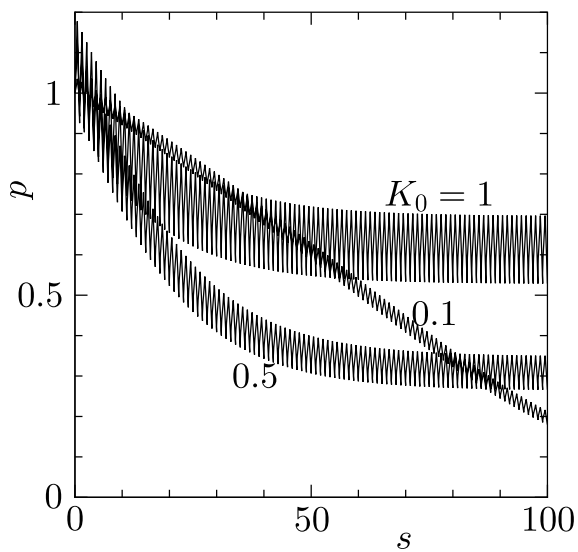

(a)

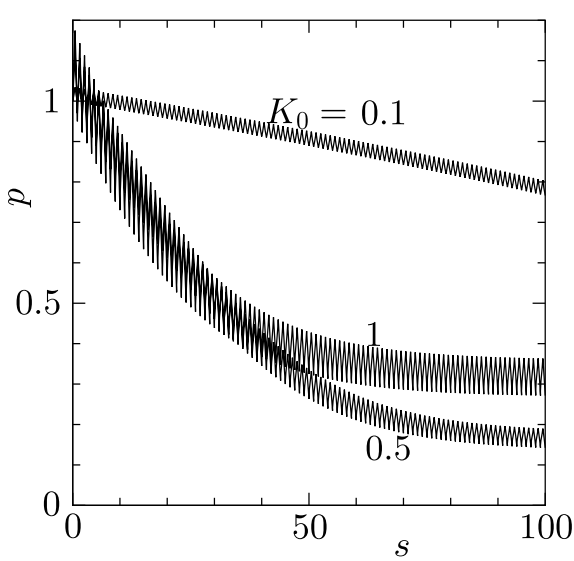

(b)

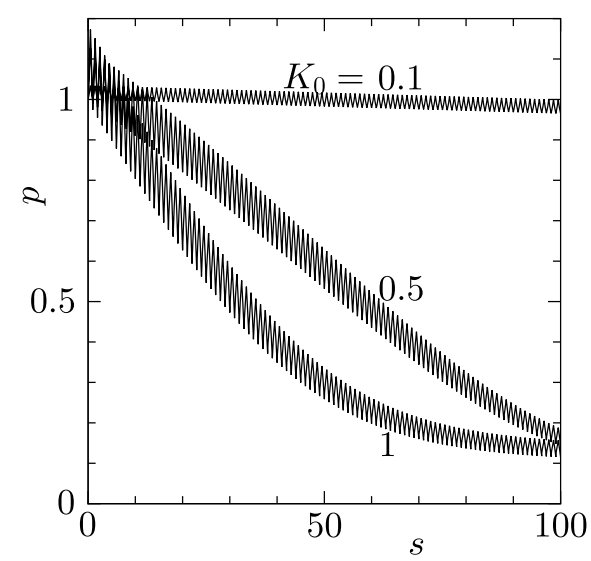

(c)

FIG. 12. Dimensionless pressure $p$ versus $s$ in the case of $N=100$. The results for $K_{0}=0.1$, 0.5 , and 1 are shown in each figure; $B=0.5, T_{H} / T_{L}=2$, and $\kappa=1$ (a), $0.5(\mathrm{~b})$, and 0.2 (c). 
RAREFIED FLOWS IN CURVED CHANNELS
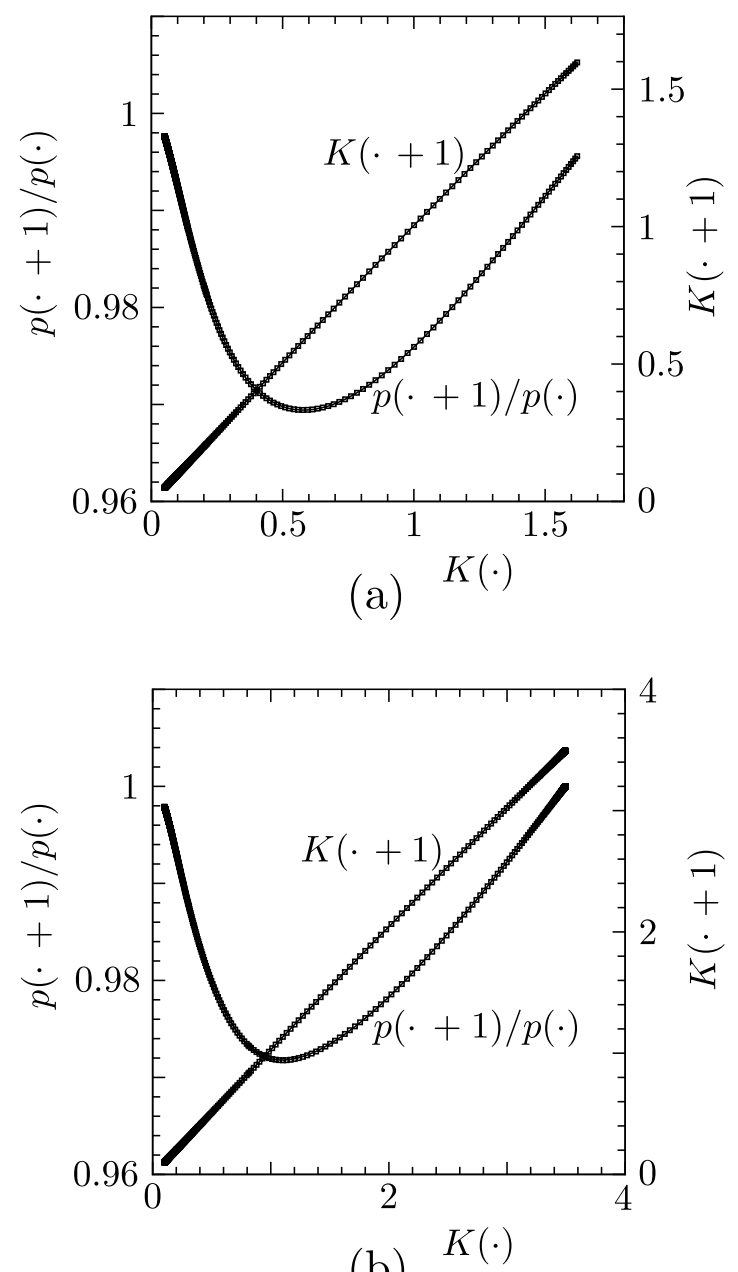

(b)

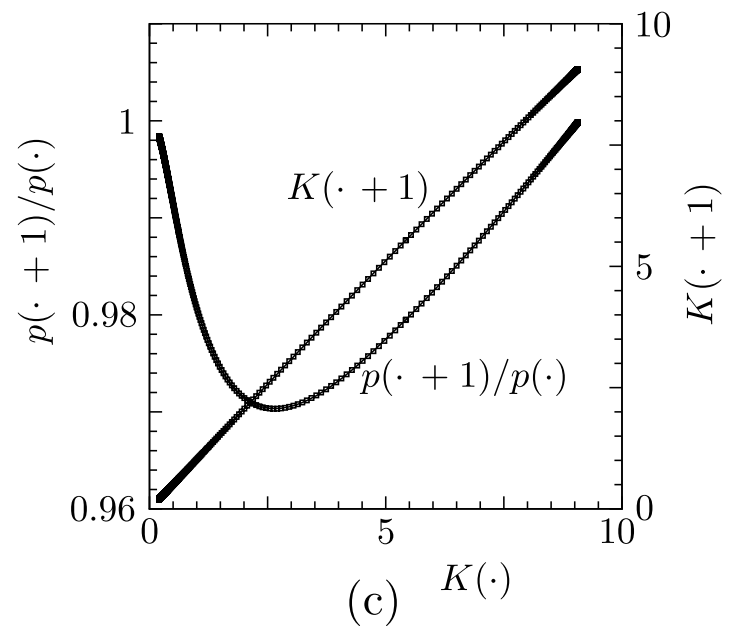

FIG. 13. $p(\cdot+1) / p(\cdot)$ versus $K(\cdot)$ together with $K(\cdot+1)$ versus $K(\cdot)$ in the case of $B=0.5$, $T_{H} / T_{L}=2$, and $\kappa=1(\mathrm{a}), 0.5(\mathrm{~b})$, and $0.2(\mathrm{c})$.

Copyright $@$ by SIAM. Unauthorized reproduction of this article is prohibited. 
For a practical application of our device, it should be equally (or even more) important to use a pipe instead of a plane channel. The behavior of the device may be slightly different in this case since, as has been noted in [5], the pipe resistance to the pressure is larger than that of a plane channel. This results in a device with a weaker flow but a stronger compression ratio. For such a system, the use of a macroscopic model is even more important, since simulations of the kinetic equation would require heavy three-dimensional computations. For the derivation of the fluid model related to this case, we point out that the construction of the database of the transport coefficients will be more complex since the auxiliary problems will have to be solved on a two-dimensional circular section instead of one-dimensional section only. This will be the subject of a future work.

Appendix A. Macroscopic quantities of Hilbert expansion. Here we give the expressions of the macroscopic quantities of the Hilbert expansion by means of $f_{(0)}$ and $f_{(1)}$ :

$$
\begin{gathered}
\rho_{(0)}=\int f_{(0)} d \boldsymbol{v} \\
\rho_{(0)} u_{s(0)}=\int v_{s} f_{(0)} d \boldsymbol{v} \\
\rho_{(0)} u_{r(0)}=\int v_{r} f_{(0)} d \boldsymbol{v}, \\
\frac{3}{2} \rho_{(0)} T_{(0)}=\int\left(\boldsymbol{v}-\boldsymbol{u}_{(0)}\right)^{2} f_{(0)} d \boldsymbol{v}, \\
\rho_{(1)}=\int f_{(1)} d \boldsymbol{v} \\
\rho_{(0)} u_{s(1)}=\int v_{s} f_{(1)} d \boldsymbol{v}-\rho_{(1)} u_{s(0)}, \\
\rho_{(0)} u_{r(1)}=\int v_{r} f_{(1)} d \boldsymbol{v}-\rho_{(1)} u_{r(0)}, \\
\frac{3}{2} \rho_{(0)} T_{(1)}=\int\left(\boldsymbol{v}-\boldsymbol{u}_{(0)}\right)^{2} f_{(1)} d \boldsymbol{v}-\frac{3}{2} \rho_{(1)} T_{(0)} .
\end{gathered}
$$

Appendix B. Computation of $\boldsymbol{f}_{(\mathbf{2})}$ and $\boldsymbol{f}_{(\mathbf{3})}$. From Lemma 2.6, the existence of the solutions $f_{(2)}$ and $f_{(3)}$ of problems (2.34) and (2.35) is proved if the right-hand sides of these equations satisfy the solvability condition (2.43).

For problem (2.34), this condition is nothing but the diffusion equation (2.21)$(2.22)$, which is satisfied by $\rho_{(0)}$ and $j_{(1)}$.

For problem (2.35) the solvability condition (2.43) must be satisfied by $\mathcal{A}^{2} f_{(1)}+$ $\mathcal{A}^{1} f_{(2)}$, since the velocity integral of the derivatives of $Q$ vanishes. This is mainly obtained by parity arguments. Since $f_{(1)}$ is odd w.r.t. $v_{s}$, so is $\mathcal{A}^{2} f_{(1)}$ (see (2.30)), and hence its velocity integral vanishes too. Finally, for $\mathcal{A}^{1} f_{(2)}$, it is sufficient to prove that $f_{(2)}$ is even, since $\mathcal{A}^{1}$ inverts the parity (see (2.29)). This can be deduced from (2.34): indeed since $f_{(1)}$ is odd, then a simple calculation shows that $D^{2} Q\left(f_{(0)}\right)\left(f_{(1)}, f_{(1)}\right)$ is zero, while $\mathcal{A}^{2} f_{(0)}$ and $\mathcal{A}^{1} f_{(1)}$ are readily seen to be even. Then point (ii) of Lemma 2.6 implies that $f_{(2)}$ is even, hence $\mathcal{A}^{1} f_{(2)}$ too.

Note that this parity of $f_{(2)}$ implies that $j_{(2)}$ is necessarily zero, which was used in section 2.2.3. 


\section{REFERENCES}

[1] K. Aoki And P. Degond, Homogenization of a flow in a periodic channel of small section, Multiscale Model. Simul., 1 (2003), pp. 304-334.

[2] K. Aoki, P. Degond, And L. Mieussens, Numerical simulations of rarefied gases in curved channels: Thermal creep, circulating flow, and pumping effect, J. Comput. Phys., submitted.

[3] K. Aoki, P. Degond, L. Mieussens, M. Nishioka, and S. Takata, Numerical simulation of a Knudsen pump using the effect of curvature of the channel, in Rarefied Gas Dynamics, M. S. Ivanov and A. K. Rebrov, eds., Siberian Branch of the Russian Academy of Sciences, Novosibirsk, Russia, 2007, pp. 1079-1084.

[4] K. Aoki, P. Degond, S. Takata, and H. Yoshida, Diffusion models for Knudsen compressors, Phys. Fluids, 19 (2007), 117103.

[5] K. Aoki, Y. Sone, S. Takata, K. Takahashi, and G. A. Bird, One-way flow of a rarefied gas induced in a circular pipe with a periodic temperature distribution, in Rarefied Gas Dynamics, Vol. 1: 22nd International Symposium, Vol. 585, T. J. Bartel and M. A. Gallis, eds., AIP, Melville, NY, 2001, pp. 940-947.

[6] H. Babovsky, On Knudsen flows within thin tubes, J. Statist. Phys., 44 (1986), pp. 865-878.

[7] H. Babovsky, C. Bardos, and T. PŁatkowski, Diffusion approximation for a Knudsen gas in a thin domain with accommodation on the boundary, Asymptotic Anal., 3 (1991), pp. 265-289.

[8] P. L. Bhatnagar, E. P. Gross, and M. Krook, A model for collision processes in gases. I. Small amplitude processes in charged and neutral one-component systems, Phys. Rev., 94 (1954), pp. 511-525.

[9] C. Cercignani, The Boltzmann Equation and Its Applications, Appl. Math. Sci. 67, SpringerVerlag, New York, 1988.

[10] C. Cercignani, Slow Rarefied Flows: Theory and Application to Micro-Electro-Mechanical Systems, Prog. Math. Phys. 41, Birkhäuser Verlag, Basel, 2006.

[11] C. Cercignani And A. Daneri, Flow of a rarefied gas between two parallel plates, J. Appl. Phys., 34 (1963), pp. 3509-3513.

[12] C.-C. Chen, I.-K. Chen, T.-P. Liu, And Y. Sone, Thermal transpiration for the linearized Boltzmann equation, Comm. Pure Appl. Math., 60 (2007), pp. 147-163.

[13] P. Degond, V. Latocha, S. Mancini, And A. Mellet, Diffusion dynamics of an electron gas confined between two plates, Methods Appl. Anal., 9 (2002), pp. 127-150.

[14] P. Degond and S. Mas-Gallic, Existence of solutions and diffusion approximation for a model Fokker-Planck equation, Transport Theory Statist. Phys., 16 (1987), pp. 589-636.

[15] P. Degond And C. Schmeiser, Macroscopic models for semiconductor heterostructures, J. Math. Phys., 39 (1998), pp. 4634-4663.

[16] V. Garzó And A. SANTos, Comparison between the Boltzmann and BGK equations for uniform shear flows, Phys. A, 213 (1995), pp. 426-434.

[17] F. Golse, Knudsen layers from a computational viewpoint, Transport Theory Statist. Phys., 21 (1992), pp. 211-236.

[18] Y. L. Han, M. Young, E. P. Muntz, And G. Shiflett, Knudsen compressor performance at low pressures, in Rarefied Gas Dynamics, M. Capitelli, ed., AIP, Melville, NY, 2005, pp. $162-167$.

[19] T. Kanki And S. IUChi, Poiseuille flow and thermal creep of a rarefied gas between parallel plates, Phys. Fluids, 16 (1973), pp. 594-599.

[20] G. Karniadakis, A. Beskok, and N. Aluru, Microflows and Nanoflows: Fundamentals and Simulation, Interdiscip. Appl. Math. 29, Springer-Verlag, New York, 2005.

[21] E. H. Kennard, Kinetic Theory of Gases, McGraw-Hill, New York, 1938.

[22] M. Knudsen, Eine revision der gleichgewichtsbedingung der gase, Annalen der Physik, 336 (1909), pp. 205-229.

[23] C. J. T. Laneryd, K. Aoki, P. Degond, and L. Mieussens, Thermal creep of a slightly rarefied gas through a channel with curved boundary, in Rarefied Gas Dynamics, M. S. Ivanov and A. K. Rebrov, eds., Siberian Branch of the Russian Academy of Sciences, Novosibirsk, Russia, 2007, pp. 1111-1116.

[24] S. K. LoyalKa, Comments on "Poiseuille flow and thermal creep of a rarefied gas between parallel plates," Phys. Fluids, 17 (1974), pp. 1053-1055.

[25] H. NiImi, Thermal creep flow of rarefied gas between two parallel plates, J. Phys. Soc. Japan, 30 (1971), pp. 572-574.

[26] T. Ohwada, Y. Sone, And K. Aoki, Numerical analysis of the Poiseuille and thermal transpiration flows between two parallel plates on the basis of the Boltzmann equation for

Copyright (C) by SIAM. Unauthorized reproduction of this article is prohibited. 
hard-sphere molecules, Phys. Fluids A, 1 (1989), pp. 2042-2049.

[27] T. Ohwada, Y. Sone, AND K. AOKI, Numerical analysis of the shear and thermal creep flows of a rarefied gas over a plane wall on the basis of the linearized Boltzmann equation for hard-sphere molecules, Phys. Fluids A, 1 (1989), pp. 1588-1599.

[28] G. Pham-Van-Diep, P. Keeley, E. P. Muntz, and D. P. Weaver, A micromechanical Knudsen compressor, in Rarefied Gas Dynamics, Vol. 1, J. Harvey and G. Lords, eds., Oxford University Press, Oxford, UK, 1995, pp. 715-721.

[29] O. ReYNOLDS, On certain dimensional properties of matter in the gaseous state. Part I and II, Phil. Trans. Roy. Soc. London, 170 (1879), pp. 727-845.

[30] F. SHARIPOV, Application of the Cercignani-Lampis scattering kernel to calculations of rarefied gas flows. I. Plane flow between two parallel plates, Eur. J. Mech. B Fluids, 21 (2002), pp. $113-123$.

[31] C. E. Siewert, The linearized Boltzmann equation: Concise and accurate solutions to basic flow problems, Z. Angew. Math. Phys., 54 (2003), pp. 273-303.

[32] Y. Sone, Thermal creep in rarefied gas, J. Phys. Soc. Japan, 21 (1966), pp. 1836-1837.

[33] Y. Sone, Flows induced by temperature fields in a rarefied gas and their ghost effect on the behavior of a gas in the continuum limit, in Annual Review of Fluid Mechanics, Annu. Rev. Fluid Mech. 32, Annual Reviews, Palo Alto, CA, 2000, pp. 779-811.

[34] Y. Sone, Kinetic Theory and Fluid Dynamics, Model. Simul. Sci. Eng. Technol., Birkhäuser Boston, Boston, MA, 2002.

[35] Y. Sone, Molecular Gas Dynamics: Theory, Techniques, and Applications, Model. Simul. Sci. Eng. Technol., Birkhäuser Boston, Boston, MA, 2007.

[36] Y. Sone and E. Itakura, Analysis of Poiseuille and thermal transpiration flows for arbitrary Knudsen numbers by a modified Knudsen number expansions and their data base, J. Vac. Soc. Jpn., 33 (1990), pp. 92-94 (in Japanese).

[37] Y. Sone, E. Itakura, and M. Handa, SOFTWARE PACKAGES of the Poiseuille Flow and the Thermal Transpiration for Rarefied Gases, http://www.users.kudpc.kyoto-u.ac. jp/ a51424/Sone/database-e.html.

[38] Y. Sone AND K. SATo, Demonstration of a one-way flow of a rarefied gas induced through a pipe without average pressure and temperature gradients, Phys. Fluids, 12 (2000), pp. $1864-1868$.

[39] Y. Sone And H. Sugimoto, Vacuum pump without a moving part and its performance, in Rarefied Gas Dynamics, E. P. Muntz and A. Ketsdever, eds., AIP, Melville, NY, 2003, pp. 1041-1046.

[40] Y. Sone And S. TAKata, Discontinuity of the velocity distribution function in a rarefied gas around a convex body and the slayer at the bottom of the Knudsen layer, Transport Theory Statist. Phys., 21 (1992), pp. 501-530.

[41] Y. Sone, Y. Waniguchi, And K. Aoki, One-way flow of a rarefied gas induced in a channel with a periodic temperature distribution, Phys. Fluids, 8 (1996), pp. 2227-2235.

[42] Y. Sone And K. Yamamoto, Flow of rarefied gas through a circular pipe, Phys. Fluids, 11 (1968), pp. 1672-1678.

[43] H. Sugimoto And Y. Sone, Numerical analysis of steady flows of a gas evaporating from its cylindrical condensed phase on the basis of kinetic theory, Phys. Fluids A, 4 (1992), pp. 419-440.

[44] S. Takata, H. Sugimoto, and S. Kosuge, Gas separation by means of the Knudsen compressor, Eur. J. Mech. B Fluids, 26 (2007), pp. 155-181.

[45] S. E. VARGo AND E. P. MunTz, Initial results from the first MEMS fabricated thermal transpiration-driven vacuum pump, in Rarefied Gas Dynamics, Vol. 1: 22nd International Symposium, Vol. 585, T. J. Bartel and M. A. Gallis, eds., AIP, Melville, NY, 2001, pp. $502-509$.

[46] P. Welander, On the temperature jump in a rarefied gas, Arkiv für Fysik, 7 (1954), pp. $507-553$. 
Copyright of Multiscale Modeling \& Simulation is the property of Society for Industrial \& Applied Mathematics and its content may not be copied or emailed to multiple sites or posted to a listserv without the copyright holder's express written permission. However, users may print. download, or email articles for individual use. 